\title{
Dynamical response of the Galileo Galilei on the ground rotor to test the equivalence principle: Theory, simulation, and experiment. I. The normal modes
}

\author{
G. L. Comandi \\ Istituto Nazionale di Fisica Nucleare (INFN), Sezione di Pisa, Largo B. Pontecorvo 3, I-56127 Pisa, Italy and \\ Department of Physics, University of Bologna, I-40I26 Bologna, Italy \\ M. L. Chiofalo \\ Scuola Normale Superiore, Piazza dei Cavalieri 7, I-56100 Pisa, Italy; Istituto Nazionale di Fisica Nucleare \\ (INFN), Sezione di Pisa, Largo B. Pontecorvo 3, I-56127 Pisa, Italy \\ R. Toncelli and D. Bramanti \\ Istituto Nazionale di Fisica Nucleare (INFN), Sezione di Pisa, Largo B. Pontecorvo 3, I-56127 Pisa, Italy \\ E. Polacco and A. M. Nobili \\ Department of Physics “E. Fermi," University of Pisa, Largo B. Pontecorvo 3, I-56127 Pisa, Italy \\ and Istituto Nazionale di Fisica Nucleare (INFN), Sezione di Pisa, Largo B. Pontecorvo, I-56127 Pisa, Italy
}

(Received 5 January 2006; accepted 16 January 2006; published online 23 March 2006)

\begin{abstract}
Recent theoretical work suggests that violation of the equivalence principle might be revealed in a measurement of the fractional differential acceleration $\eta$ between two test bodies-of different compositions, falling in the gravitational field of a source mass-if the measurement is made to the level of $\eta \simeq 10^{-13}$ or better. This being within the reach of ground based experiments gives them a new impetus. However, while slowly rotating torsion balances in ground laboratories are close to reaching this level, only an experiment performed in a low orbit around the Earth is likely to provide a much better accuracy. We report on the progress made with the "Galileo Galilei on the ground" (GGG) experiment, which aims to compete with torsion balances using an instrument design also capable of being converted into a much higher sensitivity space test. In the present and following articles (Part I and Part II), we demonstrate that the dynamical response of the GGG differential accelerometer set into supercritical rotation-in particular, its normal modes (Part I) and rejection of common mode effects (Part II) — can be predicted by means of a simple but effective model that embodies all the relevant physics. Analytical solutions are obtained under special limits, which provide the theoretical understanding. A simulation environment is set up, obtaining a quantitative agreement with the available experimental data on the frequencies of the normal modes and on the whirling behavior. This is a needed and reliable tool for controlling and separating perturbative effects from the expected signal, as well as for planning the optimization of the apparatus. (C) 2006 American Institute of Physics. [DOI: 10.1063/1.2173075]
\end{abstract}

\section{INTRODUCTION}

Experimental tests of the equivalence principle (EP) are of seminal relevance as probes of general relativity. The equivalence principle is tested by observing its consequence, namely, the universality of free fall, whereby in a gravitational field all bodies fall with the same acceleration regardless of their mass and composition. They therefore require two masses of different compositions, falling in the field of another "source" mass and a readout system to detect their motions relative to one another. An EP violation would result in a differential displacement of the masses in the direction of the source mass, which cannot be explained on the basis of known, classical phenomena (e.g., tidal effects).

The landmark experiment by Eötvös et al. ${ }^{1}$ has established that a torsion balance is most well suited for the ground tests of the EP, thanks to its inherently differential nature. With the test masses suspended on a torsion balance they improved previous pendulum experiments by almost four-orders of magnitude, showing no violation for $\eta$ larger than a few $10^{-9} .{ }^{1}$ Several decades later, by exploiting the $24 \mathrm{~h}$ modulation of the signal in the gravitational field of the Sun, torsion balance tests have improved to $10^{-11}$ (Ref. 2) and then to $10^{-12}$. $^{3}$ More recently, systematic and very careful tests carried out by $\mathrm{Su}$ et $a .^{4}{ }^{4}$ and Adelberger et al. ${ }^{5}$ using rotating torsion balances have provided even more firm evidence that no violation occurs to the level of $10^{-12}$.

The relevant theoretical question for equivalence principle tests is at which accuracy level a violation, if any, is to be expected? In an earlier work by Damour and Polyakov, based on string theory and the existence of the dilaton, ${ }^{6} \eta$ values at which a violation might be observed have been determined to be in the range $10^{-18}<\eta<10^{-13}$. Fischbach et $a .^{7}$ have derived a nonperturbative rigorous result, according to which a violation must occur at the level of $\eta$ $\simeq 10^{-17}$, due to the coupling between gravity and processes of $\nu-\bar{\nu}$ exchange which should differently affect masses with 
different nuclei. More recent work ${ }^{8}$ suggests, in a new theoretical framework for the dilaton, that a violation might occur already at the level of $\eta \simeq 10^{-12}-10^{-13}$, depending on the composition of the masses.

While an $\eta \simeq 10^{-13}$, and perhaps smaller, should be accessible with rotating torsion balance experiments on the ground, a sensitivity as high as $\eta \simeq 10^{-17}$ could be achieved only by an experiment flying in a low Earth orbit, where the driving acceleration is up to three orders of magnitude larger. Specific instruments have been designed to carry out such an experiment in space: satellite test of the equivalence principle (STEP) microscope, and "Galileo Galilei" (GG) ${ }^{9-12}$ They share two features: that the test masses are concentric cylinders and that rotation of the spacecraft provides signal modulation at frequencies higher than the orbital one.

GG is peculiar in that it spins around the symmetry axis and is sensitive to the relative displacements in the plane perpendicular to it: the cylindrical symmetry of the whole system and rotation around the symmetry axis allow a passive attitude stabilization of the spacecraft with no need of a motor after an initial spin up to the nominal frequency (typically $2 \mathrm{~Hz}$ ). The planar (instead of linear) sensitivity of the instrument is also a crucial feature for allowing us to rotate at supercritical speeds, i.e., faster than the natural frequencies of the system. Faster rotation means modulation of the signal at higher frequency and therefore a reduced $1 / f$ noise (for $1 / f$ noise see, e.g., the website maintained by $\mathrm{Li}^{13}$ ). GG differs from the other proposed space experiments also in that the test masses are suspended mechanically. We find that in the absence of weight, as it is the case in space, mechanical suspensions too can provide extremely weak coupling, with the additional advantage to electrically ground the test masses.

The GG design naturally allows us to build and test a full scale $1 \mathrm{~g}$ version of the apparatus: by suspending the instrument on a rotating platform through its spin/symmetry axis, the sensitive plane lies in the horizontal plane of the laboratory where a component of an EP violation signal might be detected, similarly to a torsion balance experiment. "Galileo Galilei on the ground" 14,15 (GGG) is primarily a prototype for testing the main novel features of the experiment proposed for flight. It is also an EP experiment in its own right aiming to compete with torsion balance tests. ${ }^{4,5}$ In this effort, motor noise, low-frequency terrain tilts, ${ }^{16}$ and tidal perturbations ${ }^{17}$ are the main issues to be addressed.

A full knowledge of the dynamical response of the GGG rotor is needed, especially in view of its condition of supercritical rotation and of its common mode rejection behavior. The theoretical understanding of the dynamical properties of the rotor, together with the construction of a full simulation facility, would allow us to predict and interpret the collected experimental data; they also provide a virtual environment for planning the experiment and optimizing its performance.

With these motivations in mind, we demonstrate that a simple but very effective mathematical model can be set up to quantitatively describe the dynamical properties of the GGG rotor. In this article (Part I), we determine the normal modes in all regimes, from subcritical to supercritical rotation, and address the issue of self-centering in the super- critical rotation. In the following article (Part II), we provide the dependence of the common mode rejection ratio on various system parameters which govern the design of the instrument.

The differential equations in the model are solved by means of a user-friendly simulation program and the numerical solutions are tested against the data available from the experiment. The physical content of the model is also discussed by means of approximate analytical solutions, which provide useful physical insight.

This article is organized as follows: Sec. II describes the main features of the experimental apparatus, Sec. III presents the dynamical model of the system, referring to specific appendixes for details, Sec. IV reports on the numerical method that we have implemented, and Sec. V gives the results obtained on the determination of the normal modes of the system, showing an excellent agreement between the theoretical predictions and experimental data. The details of the calculations are contained in two appendixes, while the third one is specifically devoted to the important concept of selfcentering. Concluding remarks are discussed in Sec. VI.

\section{THE GGG ROTOR: OVERVIEW OF THE EXPERIMENT}

GGG is a rotating differential accelerometer operated in a vacuum chamber (see Fig. 1). It is made of two concentric hollow test cylinders, $10 \mathrm{~kg}$ each, weakly coupled by means of a vertical arm-a tube located along the axis of the cylinders - to form a vertical beam balance (from now on we shall always omit the term "hollow" when referring to the test cylinders). The coupling arm is suspended at its midpoint from a rotating vertical shaft in the shape of a tube enclosing it (see Figs. 1 and 2, right hand side). A total of three suspensions are needed (drawn in red in Fig. 1): a central one (see Fig. 2, left hand side) to suspend the coupling arm from the rotating shaft and one for each test cylinder to suspend each of them from the top and bottom ends of the vertical coupling arm.

The suspensions are cardanic laminar suspensions manufactured in $\mathrm{CuBe}$ which are stiff in the axial direction $\hat{Z}$, against local gravity, and soft in the plane $\hat{X}-\hat{Y}$ orthogonal to the axis so that the geometry is naturally two dimensional, the horizontal plane being sensitive to differential accelerations acting between the test cylinders. In the normal operation mode, the modulation of such a signal is provided by setting the whole system in rotation around the vertical axis in the supercritical regime, namely, at frequencies $\nu_{s}$ larger than the natural differential frequencies of the rotor, typically $\nu_{s}>1.5 \mathrm{~Hz}$. The differential character of the instrument is strengthened by two differential readout systems made of four capacitance plates (indicated as IP, internal plates, in Fig. 1) located in between the test cylinders and which are part of two capacitance bridges in two orthogonal directions of the sensitive plane. 


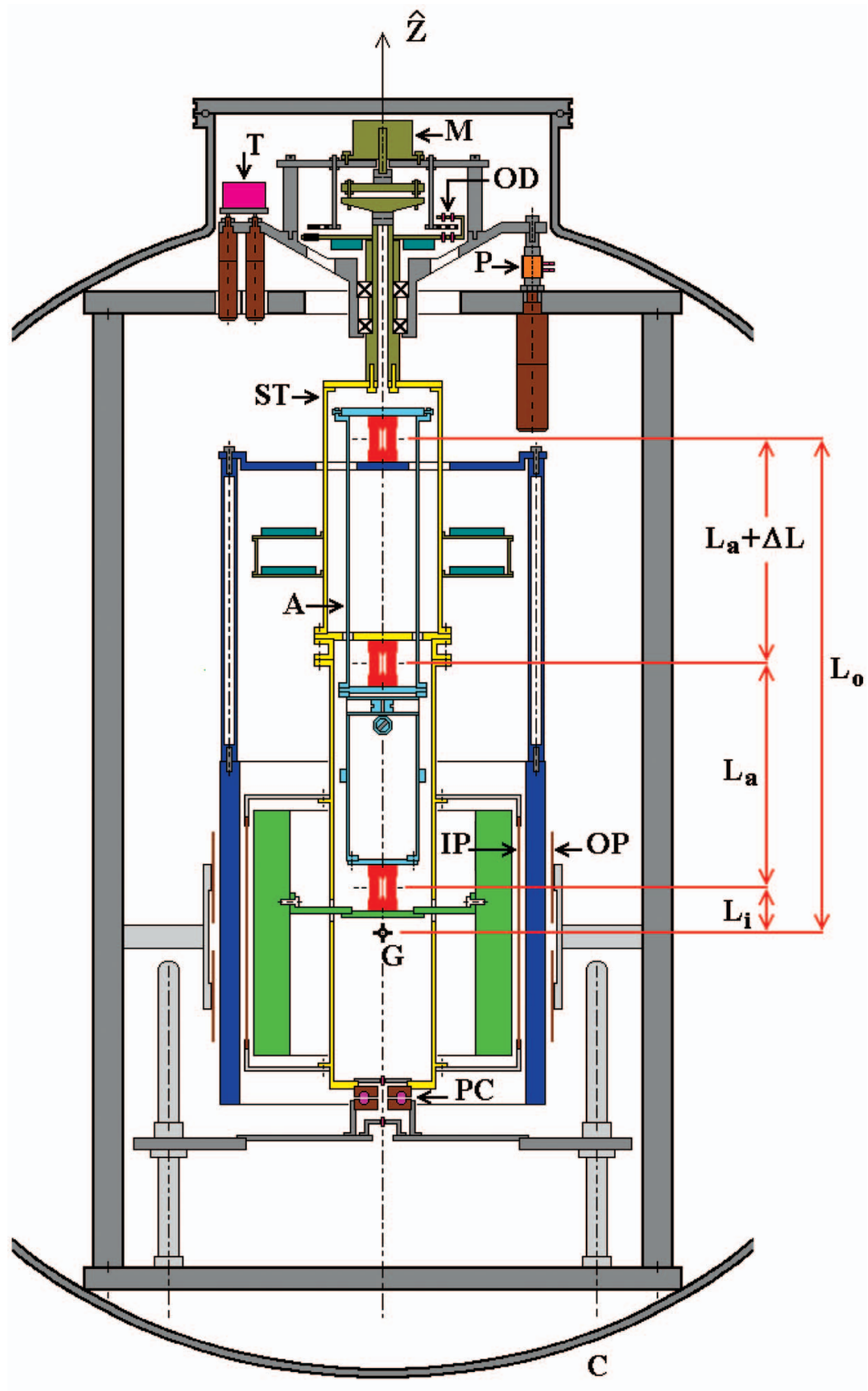

FIG. 1. (Color) Section through the spin axis $\hat{Z}$ of the differential accelerometer inside the vacuum chamber. $C$, vacuum chamber; $M$, motor; $\mathrm{OD}$, optical device (see Sec. II B); x, ball bearings; ST, suspension tube; $A$, coupling (balance) arm, located inside the suspension tube, with its three laminar cardanic suspensions (in red); and $G$, center of mass of the two cylinder's system (in blue the outer cylinder, in green the inner one, $10 \mathrm{~kg}$ each). IP are the internal capacitance plates of the differential motion detector (Sec. II B), OP are the outer ones for whirl control (Sec. II C 2), and PC is the contactless inductive power coupler providing power to the electronics inside the rotor. The relevant distances, $L_{i}$ and $L_{o}$, of the centers of mass of the inner and outer bodies from their suspension points are also sketched, along with the arm length $2 L_{a}+\Delta L . T$ and $P$, at the top of the rotor, are the tiltmeter and three-PZTs (at $120^{\circ}$ from one another-only one shown) for automated control of low frequency terrain tilts. The drawing is to scale and the inner diameter of the vacuum chamber is $1 \mathrm{~m}$.

\section{A. Description of the mechanical structure of the apparatus}

The GGG apparatus is schematically presented in Fig. 1, where a section through the spin-symmetry axis $\hat{Z}$ is shown inside the vacuum chamber $C$. At the top center of the frame is the motor $M$ whose shaft is connected to the suspension tube of the rotor ST (drawn in yellow) by means of an appropriate motor-rotor joint and turns in the vertical direction inside ball bearings, indicated by $\mathbf{x}$ symbols in the figure. From the suspension tube ST rotation is then transmitted to a tube located inside it which constitutes the vertical beam of the balance (also referred to as the coupling arm, Fig. 2, right hand side), the connection between the two being provided at the midpoint of the arm by the central laminar cardanic suspension (see Fig. 2, left hand side).

The coupling arm in its turn transmits rotation to both the test cylinders, since they are suspended (by means of two laminar cardanic suspensions similar to the central one) from its two ends. More precisely, the inner test cylinder (shown in green) is suspended from the bottom of the coupling arm at a distance $L_{i}$ from the cylinder's center of mass, while the outer one (shown in blue) is suspended from the top of the coupling arm, at a distance $L_{o}$ from the cylinder's center of mass. In Fig. 1 the three suspensions are drawn in red. It is apparent that the central suspension carries the whole weight of the rotor, mostly the weight of the two test cylinders (10 kg each) plus the small weight of the coupling arm. It is worth noting that the metallic suspensions provide a passive electrostatic discharging of the test masses.

In this way, the symmetry of the whole apparatus is cylindrical, its axis being both the vertical beam of the balance and the axis of rotation, the balance is sensitive in the horizontal plane, and the test masses are concentric. 


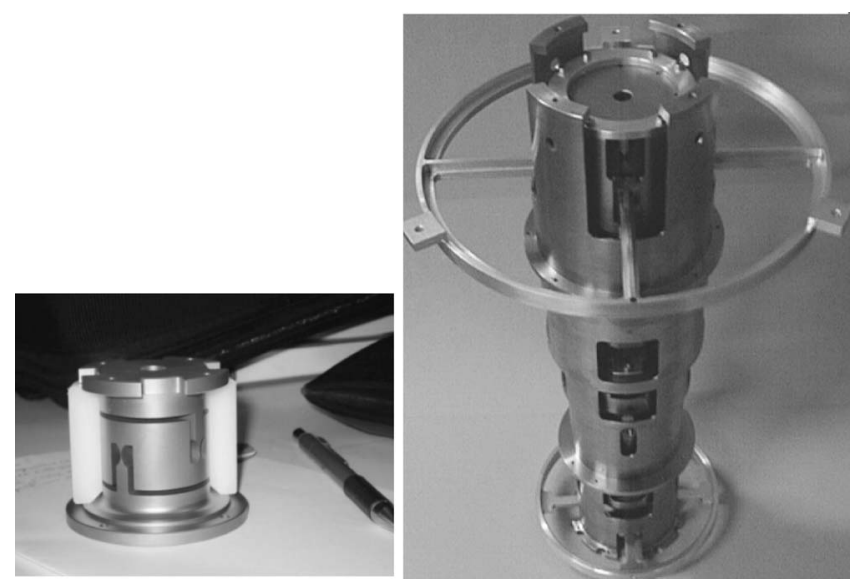

FIG. 2. Left hand side: the central laminar cardanic suspension of the GGG rotor, located at the midpoint to the coupling arm in order to suspend it from the suspension tube (shaft). Right hand side: the coupling arm inside the suspension tube (shaft) as seen from the top. Two cardanic laminar suspensions are located at its top and bottom ends. They suspend the test cylinders (not shown here) through two metal rings. The dimensions of the rings depend on the dimensions of the concentric cylinders, which have equal mass $(10 \mathrm{~kg})$ and therefore different sizes. The top and bottom rings refer to outer and inner test cylinders, respectively.

\section{B. The differential motion detector system}

The differential motion detector (DMD) reflects the cylindrical symmetry of the system and is composed of the following three parts.

(1) Two capacitance plates IP working as $\operatorname{DMD}(x)$ sensors (drawn as vertical lines in between the cylinders in Fig. 1) are located halfway in between the test cylinders in correspondence to the $X$ direction with a clear gap of $5 \mathrm{~mm}$ on either side and are connected to the suspension tube by means of an insulating frame. A similar pair of capacitances forming a $\operatorname{DMD}(y)$ is placed in the $Y$ direction. A voltage signal is applied to each capacitance bridge in order to shift the signal of interest to a highfrequency band with reduced $1 / f$ noise (with phase locked detection). The filtered signal is digitized by an analog-to-digital converter (ADC) before transmission to the nonrotating (laboratory) frame. Calibration and balancing of the capacitance bridge are performed by means of the procedures outlined in Ref. 14. The best sensitivity achieved in bench tests corresponds to mechanical displacements of $5 \mathrm{pm}$ in $1 \mathrm{~s}$ of integration time. ${ }^{11,14}$ Presently, the sensitivity of the readout system during normal operation is $\simeq 10^{-9} \mathrm{~m}$.

(2) An optical device OD located below the motor and above the ball bearings, utilizing a disk with 32 holes and an infrared emitter-detector pair, provides a reference signal for the angular position of the rotor. The reference signal is combined with the $X$ and $Y$ channel data from the DMD and encoded into RS232 format for transmission to a computer. Then a second emitterdetector pair located at the very bottom of the rotor (using a hole along the axis of the power coupler PC, see Fig. 1) transmits the digital signal from the rotor to the nonrotating frame from where it is taken out of the vacuum chamber through electrical feedthroughs.
(3) An annular disk, in two semicircular parts, is mounted around the upper half of the suspension tube and contains the two capacitance-bridge circuits and their preamplifiers (see Fig. 1). The necessary electronics to demodulate the signal and convert it from an analog to digital form as well as the drivers for the optical emitter are also located here.

\section{Principle of operation}

For detecting an EP violation signal the instrument relies on its sensitivity to the relative displacement of the two test masses, which in the final design will be made of different materials. An acceleration in the horizontal plane of the laboratory acting differently between the test cylinders gives rise to a relative displacement of the two in the direction of the acceleration. This displacement unbalances the capacitance bridges and gives rise to an electric voltage proportional to it.

A modulation of the displacement, as seen by the capacitance plates, is achieved by setting the whole system in rotation around the vertical axis of symmetry passing through the shaft, as shown in Fig. 1. Note that the signal modulation obtained in this way does not affect the centers of mass of the test cylinders; hence, it does not affect their relative displacement, which is the physical quantity measured in the experiment. As a result, this type of modulation reduces the noise but not the signal.

In fact, this signal modulation could be achieved by keeping the test cylinders stationary and rotating only the capacitance plates (located in between the two, indicated as IP in Fig. 1) which form the differential motion detector system described above. However, by rotating the test cylinders together with the capacitors, any irregularity in their mass distribution averages out; moreover, the supercritical regime can be exploited to reduce the rotation noise for all parts of the apparatus (see Sec. II C 2 and Appendix C). As for the experiment in space, the rotation of the whole spacecraft has two more very important advantages. In the first place, it eliminates the need for motor and ball bearings altogether, which are a considerable source of noise in the ground experiment. Secondly, by rotating around the axis of maximum moment of inertia, the spacecraft is passively stabilized, thus reducing its weight, cost, and complexity, as well as disturbances on the EP experiment.

An EP violation signal in the gravitational field of either the Earth or the Sun would have a component in the horizontal plane of the laboratory which could be detected by the instrument. Since the test bodies are rotors suspended on the Earth and the Earth rotates around its axis, this diurnal rotation gives rise to large gyroscopic effects on the test bodies resulting in a nonzero differential acceleration which would mask an EP violation signal in the field of the Earth itself. The measurements of such gyroscopic effects have been reported in Ref. 14 (Sec. V, Fig. 12). The instrument-in this ground based version-is therefore used for two purposes: (i) to establish its sensitivity as a prototype of the flight instrument, namely, for an expected signal at the orbital frequency of the satellite $\left(\simeq 1.75 \times 10^{-4} \mathrm{~Hz}\right.$, i.e., about $1 \frac{1}{2} \mathrm{~h}$ period, at an Earth orbiting altitude of $\simeq 520 \mathrm{~km}$ ) and (ii) to 
look for an EP violation in the gravitational field of the Sun, in which case the signature of the signal (see Ref. 12, Sec. II) would have a dominant Fourier component of a $24 \mathrm{~h}$ period due to the diurnal rotation of the Earth.

\section{Differential character and common mode rejection}

The differential character of the whole instrument, namely, its capability to reject accelerations which are common to both test masses, is in principle ensured by the geometry and mounting of the test masses. It is further augmented by the differential nature of the DMD system.

The sensitivity of the instrument to differential accelerations of the test masses depends on the softness of the laminar suspensions and on the uniform distribution of mass around the spin axis. Soft suspensions and a good balancing of the rotor provide long natural periods for differential oscillations of the test masses relative to each other, giving rise to larger relative displacements between the two and, in turn, to stronger output voltage signals.

The tuning of the natural differential period $T_{D}$ of the test cylinders is made possible by changing a moment arm in the beam balance. This is accomplished by moving a small solid ring mounted at the lower end of the balance (coupling) arm. Moving this ring vertically along the arm, in the $\hat{Z}$ direction, displaces the center of mass of the balance arm from its suspension point by a quantity $\Delta L$. If $\Delta L=0$ the center of mass of the balance arm is coincident with its suspension point. $\Delta L$ can be adjusted to be either slightly positive or negative, resulting in a longer or shorter $T_{D}$. However, there is a maximum positive value that $\Delta L$ can assume before the system becomes unstable [see Eq. (39) below].

Asymmetric distribution of mass of the rotor in the horizontal plane, resulting in a nonzero inclination of the coupling arm in the rotating reference frame, may also be corrected by two small masses mounted inside the coupling arm itself, one of which is movable in the $X$ direction and the other in the $Y$.

The tilt of the spin axis with respect to the nonrotating laboratory frame is controlled by $3 \mu \mathrm{m}$ screws which support the plate on which the rotor shaft is mounted. In addition the tilt can be finely adjusted using piezoelectric actuators $(P)$ attached to the tips of the micrometer screws (see Fig. 1).

(ii) As to the DMD system, a nonzero off centering of the capacitor plates IP located in between the test cylinders-measured by the ratio $(a-b) / a$ where $a(b)$ is the nominal gap between the inner (outer) mass and any one of the capacitance plates-would make a common mode displacement $\Delta x_{C}$ of the test masses to produce a differential output signal in addition to that produced by a real differential displacement $\Delta x_{D}$. The larger this off centering, the larger the fraction of the common mode displacement which is turned into a "fake" differential signal, i.e., which contributes to the total unbalance $\Delta C$ of the capacitance bridge ${ }^{11}$ (GG
Phase A Report, Sec. 2.1.3) from the original capacitance value $C_{o}$,

$$
\frac{\Delta C}{2 C_{0}} \simeq \frac{a-b}{a^{2}} \Delta x_{C}-\frac{1}{a} \Delta x_{D} .
$$

\section{Signal modulation and whirl motions}

Signal modulation in testing the equivalence principle has been first proposed in Ref. 2 in order to improve the experiments of Eötvös et al.. By referring to the Sun rather than the Earth as the source mass of the gravitational field, the diurnal rotation of the Earth itself on which the test masses are suspended provides a $24 \mathrm{~h}$ modulation with no need to rotate the experimental apparatus, an operation which gives rise to relevant disturbances in such small force experiments. However, higher modulation frequencies are desirable in order to reduce the $1 / f$ noise and, in fact, excellent results have been obtained by Refs. 4 and 5 with torsion balances placed on a turntable rotating faster than the Earth. In GGG we try to spin the test masses much faster, at frequencies (typically a few hertz) higher than the natural frequencies $\nu_{n}$ of the system, a condition known as supercritical rotation.

The GGG apparatus has three natural frequencies. The differential frequency $\nu_{D}$ of the oscillations of the test bodies relative to one another and two common mode frequencies, $\nu_{C 1}$ and $\nu_{C 2}$, of both test masses together. In the GGG setting reported here their values are $\nu_{D}=0.09 \mathrm{~Hz}, \nu_{C_{1}}=0.91 \mathrm{~Hz}$, and $\nu_{C_{2}}=1.26 \mathrm{~Hz}$.

It is well known ${ }^{18-20}$ that in supercritical rotation the masses are able to self-center and greatly reduce the original offsets of their centers of mass with respect to their own rotation axes. Any initial offset, which inevitably results from construction and mounting errors, is, in fact, reduced by a factor $\left(\nu_{D} / \nu_{s}\right)^{2}$. Such self-centering is a very essential requirement when using fast rotating macroscopic test bodies for the purpose of detecting the effects of extremely small forces between them.

It is also well known that in supercritical rotation, dissipation in the system gives rise to destabilizing whirl motions at frequencies $\nu_{w}$ equal (or close) to the natural frequencies of the system, whose amplitude increases with time at a rate $1 / \tau_{w}=\pi \nu_{w} / \mathcal{Q}\left(\nu_{s}\right)$ scaling as the whirl frequency $\nu_{w}$ and the inverse of the quality factor $\mathcal{Q}$ at the spin frequency $\nu_{s}{ }^{20-22}$

Whirls can be stabilized by passive and active methods. Passive stabilization is typically used in engineering applications of supercritical rotors, but it produces too large disturbances for our purposes. We have used a passive damper in the past only to stabilize the rotor during resonance crossing (see Ref. 14, Sec. III). With the current improved apparatus, damping at resonance crossing is no longer needed. A much finer whirl stabilization can be performed actively by means of eight small capacitance sensors/actuators (indicated as OP, outer plates, in Fig. 1) placed close to the outside surface of the outer test cylinder, four of them used as sensors and four as actuators in two orthogonal directions of the horizontal plane. ${ }^{15}$ In the GGG experiment performed at supercritical speed the relevant $\mathcal{Q}$ value is determined by losses due to 


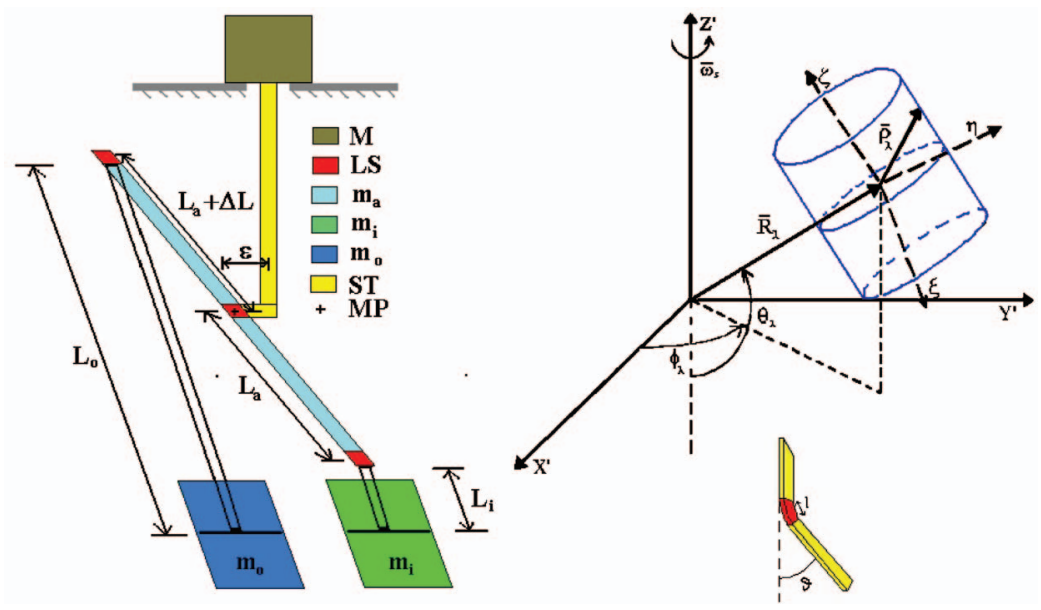

FIG. 3. (Color) Minimal model for the real instrument sketched in Fig. 1 (see text for details). On the left hand side the various parts are drawn with the same colors and labels as in Fig. 1. Here the midpoint of the coupling arm is indicated as MP. $L_{a}, L_{o}$, and $L_{i}$ refer to the dimensions of the coupling arm and the outer mass and inner mass suspension arms, respectively. $\hat{L}_{a}, \hat{L}_{o}$, and $\hat{L}_{i}$ are the unit vectors of the corresponding beams. The offset vector $\boldsymbol{\epsilon}$, due to construction and mounting imperfections, is also indicated. On the right hand side we sketch one of the cylinders in the rotating reference frame $\left\{X^{\prime} Y^{\prime} Z^{\prime}\right\}$, showing its principal axes of inertia $\{\xi, \eta, \zeta\}$, the position vector $\mathbf{R}_{\lambda}(\lambda=a, o, i)$ of its center of mass, and the angles $\theta_{\lambda}, \phi_{\lambda}$, which are not the usual Euler angles, as discussed in the text. Below this figure, the small one to the right shows a typical deformation of one of the laminar suspensions of length $l$, for instance, the central one. None of these figures is to scale. deformations of the laminar suspensions at the spin frequency. Experimental measurements of $\mathcal{Q}$ are reported in Ref. 14 and, more recently, in Ref. 23.

\section{THE MODEL}

Having described the real instrument, we are now in a position to outline the minimal model used to describe its dynamical behavior. Figure 3 displays a schematic representation of the model in the reference frame $\left\{X^{\prime} Y^{\prime} Z^{\prime}\right\}$ rotating with the shaft at an angular velocity $\omega_{s}=2 \pi \nu_{s}$ around the $Z^{\prime}$ axis $\left(\boldsymbol{\omega}_{s}=\omega_{s} \hat{Z}^{\prime}\right)$. The relevant parts of the instrument depicted in Fig. 1 are sketched in Fig. 3 with the same colors. The coupling arm, with mass $m_{a}$ (drawn in cyan as in Fig. 1) and length $2 L_{a}+\Delta L$, is suspended at its midpoint MP from the rotating shaft and suspension tube ST (yellow) by means of the central laminar suspension LS (red) with elastic constant $K$. The vector $\boldsymbol{\epsilon}$ is the offset of the arm center of mass from the axis, which is unavoidable because of construction and mounting errors. Variations of $\Delta L$, as we have already discussed, produce a change of the mass distribution, hence of the natural differential period of the test masses, $T_{D}$. Here, and with no loss of generality, $\boldsymbol{\epsilon}$ is placed along the $X^{\prime}$ axis.

The outer test cylinder, of mass $m_{o}$ (blue), is suspended from the top of the coupling arm by means of the laminar suspension with elastic constant $K_{o}$ and its center of mass is at a distance $L_{o}$ from the suspension. In a similar manner, the inner test mass $m_{i}$ (green) is suspended from the bottom of the arm, $K_{i}$ and $L_{i}$ being the corresponding parameters. From now on, the label $\lambda=i, o, a$ will be used to refer to the parameters of the inner mass, outer mass, and coupling arm, respectively. The three bodies have moments of inertia $I_{\lambda \xi}$ $=I_{\lambda \eta}=m_{\lambda}\left(3 R_{\lambda I}^{2}+3 R_{\lambda E}^{2}+R_{\lambda H}^{2}\right) / 12$ and $I_{\lambda \zeta}=m_{\lambda}\left(R_{\lambda I}^{2}+R_{\lambda E}^{2}\right) / 2$ along their principal axes $(\xi, \eta$, and $\zeta), R_{\lambda I}$ and $R_{\lambda E}$ being the internal and external radii of the cylinder $\lambda$, and $R_{\lambda H}$ its height.

The laminar suspensions have length $l$, the central one is slightly stiffer than the other two and we assume $K_{i}=K_{o}$ $\neq K$. In a refined version of the model, and whenever specified, we also consider an anisotropic central suspension by introducing the parameter $\Lambda$ such that $K_{Y^{\prime}} \equiv \Lambda K_{X^{\prime}}$.

By defining the unit vector $\hat{L}_{a}$ of the coupling arm as pointing from its midpoint towards the bottom suspension, and the unit vectors $\hat{L}_{o}$ and $\hat{L}_{i}$ of the test cylinders each pointing from the suspension to the center of mass of the body (see Fig. 3), the corresponding position vectors in the rotating reference frame $\left\{X^{\prime} Y^{\prime} Z^{\prime}\right\}$ of Fig. 3 are

$$
\begin{aligned}
& \mathbf{R}_{a}=\boldsymbol{\epsilon}-0.5 \Delta L \hat{L}_{a}, \\
& \mathbf{R}_{o}=\boldsymbol{\epsilon}-\left(L_{a}+\Delta L\right) \hat{L}_{a}+L_{o} \hat{L}_{o}, \\
& \mathbf{R}_{i}=\boldsymbol{\epsilon}+L_{a} \hat{L}_{a}+L_{i} \hat{L}_{i} .
\end{aligned}
$$

\section{A. The Lagrangian}

The Lagrangian $\mathcal{L}$ in the rotating reference frame $\left\{X^{\prime} Y^{\prime} Z^{\prime}\right\}$ can be written as

$$
\mathcal{L}=\mathcal{T}-\mathcal{U}
$$

where the kinetic term can be very generally written as

$$
\mathcal{T}=\frac{1}{2} \sum_{\lambda=a, o, i} \int_{\tau_{\lambda}} \boldsymbol{v}_{\lambda}^{2} d m_{\lambda},
$$

after defining the velocity $\boldsymbol{v}_{\lambda}$ of the mass element $d m_{\lambda}$ in body $\lambda$ with volume $\tau_{\lambda}$. Then, $\mathcal{U}$ includes the potential energies associated with gravity and with the elastic forces, namely,

$$
\mathcal{U}=U_{g}+U_{\mathrm{el}}
$$

where

$$
\begin{aligned}
U_{g}= & \sum_{\lambda=a, o, i}-m_{\lambda} \mathbf{g} \cdot \mathbf{R}_{\lambda} \quad \text { with } \mathbf{g} \equiv-g \hat{Z}^{\prime}, \\
U_{\mathrm{el}}= & \sum_{\lambda=o, i} \frac{1}{2} K_{\lambda} l^{2}\left|\hat{R}_{a} \times \hat{R}_{\lambda}\right|^{2} \\
& +\frac{1}{2} L_{a}^{2}\left(K_{X^{\prime}}\left|\hat{R}_{a} \dot{\hat{X}}^{\prime}\right|^{2}\right. \\
& \left.+K_{Y^{\prime}}\left|\hat{R}_{a} \dot{Y}^{\prime}\right|^{2}\right) .
\end{aligned}
$$

For the expression of $U_{\mathrm{el}}$ we refer to the small figure at the bottom right of Fig. 3, sketching the laminar suspension and its orientation.

We proceed along the main steps to derive the operational expression for $\mathcal{L}$. The bodies are rotating around their 
own axis with angular velocity $\omega_{s}$ in a reference frame which is rotating as well, as sketched in Fig. 3, right hand side. Thus, we define as $\boldsymbol{\Omega}_{\lambda}$ the angular-velocity vector of the element $d m_{\lambda}$ in the $\left\{X^{\prime} Y^{\prime} Z^{\prime}\right\}$ frame and $\boldsymbol{\omega}_{s}=\omega_{s} \hat{Z}^{\prime}$, so that

$$
\boldsymbol{v}_{\lambda}=\mathbf{V}_{\lambda}+\boldsymbol{\Omega}_{\lambda} \times \mathbf{r}_{\lambda}=\mathbf{V}_{\lambda}+\boldsymbol{\Omega}_{\lambda} \times\left(\mathbf{R}_{\lambda}+\boldsymbol{\rho}_{\lambda}\right),
$$

where $\mathbf{V}_{\lambda}$ is the velocity of the center of mass of body $\lambda$ and $\mathbf{r}_{\lambda}$ is the vector pointing to the element $d m_{\lambda}$, composed by $\mathbf{R}_{\lambda}$ and $\boldsymbol{\rho}_{\lambda}$, as drawn in Fig. 3. By inserting Eq. (8) into Eq. (4), we can write $\mathcal{T}$ as

$$
\mathcal{T}=T_{\text {kin }}+T_{\text {cor }}+U_{\text {cor }}+U_{c},
$$

where the only nonzero terms are (see Appendix A for details)

$$
\begin{aligned}
& T_{\text {kin }}=\frac{1}{2} \sum_{\lambda}\left(m_{\lambda} V_{\lambda}^{2}+\sum_{\alpha=\xi, \eta, \zeta} I_{\lambda \alpha \alpha} \Omega_{\alpha}^{2}\right), \\
& T_{\text {cor }}+U_{\text {cor }}=\sum_{\lambda} m_{\lambda} \mathbf{V}_{\lambda} \cdot\left(\boldsymbol{\omega}_{s} \times \mathbf{R}_{\lambda}\right) \\
& \quad+\sum_{\lambda} \int_{\tau_{\lambda}}\left(\boldsymbol{\Omega}_{\lambda} \times \boldsymbol{\rho}_{\lambda}\right) \cdot\left(\boldsymbol{\omega}_{s} \times \boldsymbol{\rho}_{\lambda}\right) d m_{\lambda}, \\
& U_{c}=\frac{1}{2} \sum_{\lambda} \int_{\tau_{\lambda}}\left[\boldsymbol{\omega}_{s} \times\left(\mathbf{R}_{\lambda}+\boldsymbol{\rho}_{\lambda}\right)\right]^{2} d m_{\lambda} .
\end{aligned}
$$

In Eq. (11) the terms coming from Coriolis forces have been split into the $U_{\text {Cor }}$ potential energy, which contains only the position vectors, and $T_{\mathrm{Cor}}$ which contains also the velocities. The centrifugal part $U_{c}$ has been indicated as a potential energy. To proceed further, we now have to specify the choice of the generalized coordinates.

\section{B. Choice of the generalized coordinates}

The GGG rotor model shown in Fig. 3 is composed of $n_{b}=3$ coupled bodies, for a total of 18 degrees of freedom. However, the central suspension prevents them from performing translational motions, thereby reducing the degrees of freedom to 9. In addition, the motor forces the three bodies to rotate at a constant angular velocity, so that the number of degrees of freedom for the model is $n=6$.

We have chosen as generalized coordinates for each body the two angles $\theta_{\lambda}$ and $\phi_{\lambda}$ (see Fig. 3, right hand side). These angles are defined slightly differently from the usual Euler angles: $\theta_{\lambda}$ is the angle between $\mathbf{R}_{\lambda}$ and the axis $-\hat{Z}^{\prime}$ and runs in the interval $[0, \pi] ; \phi_{\lambda}$ is the angle from the $\hat{X}^{\prime}$ axis to the projection of $\mathbf{R}_{\lambda}$ on the $X^{\prime} Y^{\prime}$ plane and runs in the interval $[0,2 \pi]$. We thus define the vector $Q$ of the generalized coordinates and the corresponding velocities $\dot{Q}$

$$
Q=\left\{q_{1}, q_{2}, q_{3}, q_{4}, q_{5}, q_{6}\right\}=\left\{\theta_{a}, \phi_{a}, \theta_{o}, \phi_{o}, \theta_{i}, \phi_{i}\right\} .
$$

With these definitions in hand, we have that

$$
\hat{L}_{a}=\left\{\sin \theta_{a} \cos \phi_{a}, \sin \theta_{a} \sin \phi_{a},-\cos \theta_{a}\right\},
$$

and similar expressions for $\hat{L}_{o}$ and $\hat{L}_{i}$. Equation (14) turns Eqs. (2) into expressions for the $\mathbf{R}_{\lambda}(Q)$ and the corresponding velocities $\mathbf{V}_{\lambda}=\dot{\mathbf{R}}_{\lambda}(Q, \dot{Q})$. We then conveniently write all the vectors in the $\left\{X^{\prime} Y^{\prime} Z^{\prime}\right\}$ reference frame in terms of their components in the $\{\xi \eta \zeta\}$ frame by means of the rotation matrix $\mathcal{M}$ (Eq. (A2)), namely, $\boldsymbol{\Omega}_{\lambda}=\overleftrightarrow{\mathcal{M}} \boldsymbol{\Omega}_{\lambda, \xi \eta \zeta}$ and $\boldsymbol{\rho}_{\lambda}=\stackrel{\leftrightarrow}{\mathcal{M}} \boldsymbol{\rho}_{\lambda, \xi \eta \zeta}$

After noting that $\boldsymbol{\Omega}_{\lambda, \xi \eta \zeta}=\left\{-\dot{\theta}_{\lambda}, \dot{\phi}_{\lambda} \sin \theta_{\lambda}, \omega_{s}\right\}$ and performing all the integrals over the three bodies, we finally obtain (Appendix A) the operative expression for $\mathcal{L}(Q, \dot{Q})$ in the rotating reference frame,

$$
\mathcal{L}(Q, \dot{Q})=T(Q, \dot{Q})-U(Q),
$$

where we have defined

$$
\begin{aligned}
& T(Q, \dot{Q}) \equiv T_{\mathrm{kin}}(Q, \dot{Q})+T_{\mathrm{Cor}}(Q, \dot{Q}), \\
& U(Q) \equiv U_{g}(Q)+U_{\mathrm{el}}(Q)-U_{\mathrm{Cor}}(Q)-U_{c}(Q) .
\end{aligned}
$$

The terms entering (15) and (17) are

$$
\begin{aligned}
T_{\text {kin }}= & \frac{1}{2} \sum_{\lambda}\left[m_{\lambda} V_{\lambda}(Q, \dot{Q})^{2}+I_{\lambda \xi}\left(\dot{\phi}_{\lambda}^{2} \sin ^{2} \theta_{\lambda}+\dot{\theta}_{\lambda}^{2}\right)\right], \\
T_{\text {Cor }}= & \sum_{\lambda} m_{\lambda} \mathbf{V}_{\lambda}(Q, \dot{Q}) \cdot\left[\boldsymbol{\omega}_{s} \times \mathbf{R}_{\lambda}(Q)\right] \\
& +\sum_{\lambda} I_{\lambda \xi} \omega_{s} \dot{\phi}_{\lambda} \sin ^{2} \theta_{\lambda}, \\
U_{\text {Cor }}= & -\sum_{\lambda} I_{\lambda \zeta} \omega_{s}^{2} \cos \theta_{\lambda},
\end{aligned}
$$

and

$$
\begin{aligned}
U_{c}= & \frac{1}{2} \sum_{\lambda} m_{\lambda}\left[\boldsymbol{\omega}_{s} \times \mathbf{R}_{\lambda}(Q)\right]^{2}+\frac{1}{2} \sum_{\lambda}\left(I_{\lambda \xi} \sin ^{2} \theta_{\lambda}\right. \\
& \left.+I_{\lambda \zeta} \cos ^{2} \theta_{\lambda}\right) \omega_{s}^{2} .
\end{aligned}
$$

To these equations we have to add the expressions (6) and (7) written in terms of $\mathbf{R}_{\lambda}(Q)$ through (2) and (14).

Equation (15) together with Eqs. (17)-(19) yield the Lagrange function of the model in Fig. 3.

\section{Equilibrium positions and second-order expansion}

During normal and successful operation of the GGG rotor only very small amplitude motions take place. The Lagrange function (15) can thus be expanded to second order in $(Q, \dot{Q})$ around the equilibrium solution $\left(Q^{0}, \dot{Q}=0\right), Q^{0}$ $=\left\{q_{1}^{0}, \ldots, q_{6}^{0}\right\}$ to derive linearized equations of motion.

In order to do this, we first determine the equilibrium positions from the equation

$$
\left.\frac{\partial U}{\partial q_{j}}\right|_{q_{j}=q_{j}^{0}}=0, \quad j=1, \ldots, n .
$$

We then use the physical assumption that during the motion, the $Q$ 's are slightly perturbed from their equilibrium values $Q^{0}$. This results in the substitutions

$$
\begin{aligned}
& Q \rightarrow Q^{0}+Q, \\
& \dot{Q} \rightarrow \dot{Q},
\end{aligned}
$$

into (15) to obtain a linearized version of the Lagrange function. $\mathcal{L}(Q, \dot{Q})$ can now be expanded to second order, namely, 


$$
\begin{aligned}
\mathcal{L}(Q, \dot{Q})= & a_{0}+\sum_{j<k}^{n} a_{j k} q_{j} q_{k}+\sum_{j<k}^{n} b_{j k} \dot{q}_{j} \dot{q}_{k}+\sum_{j, k=1}^{n} c_{j k} q_{j} \dot{q}_{k} \\
& +\sum_{j=1}^{n} d_{j} \dot{q}_{j}+O\left(q_{j}, \dot{q}_{k}\right)^{4},
\end{aligned}
$$

where we remark that now the $q_{j}$ 's are small according to the substitutions (21) and (22), and that the linear terms have canceled out because of (20). The matrix coefficients $a_{j k}, b_{j k}$, and $c_{j k}$ are known functions of the $Q^{0}$ and of the governing parameters of the system and, in general, are to be numerically evaluated.

\section{Linearized equations of motion}

The equations of motion in terms of the known $a_{j k}, b_{j k}$, and $c_{j k}$ coefficients are

$$
\frac{d}{d t} \frac{\partial \mathcal{L}}{\partial \dot{q}_{j}}-\frac{\partial \mathcal{L}}{\partial q_{j}}=\mathcal{F}_{j}, \quad j=1, \ldots, n,
$$

where we have introduced the generalized forces

$$
\mathcal{F}_{j}=\sum_{d=1}^{3} \mathbf{F}_{\lambda d} \frac{\partial \mathbf{R}_{\lambda d}}{\partial q_{j}},
$$

starting from the Cartesian components $\mathbf{F}_{\lambda d}$ of the forces acting on each body. The $\mathcal{F}_{j}$ are to be consistently expanded to first order, namely,

$$
\mathcal{F}_{j}=\sum_{k=1}^{6} \alpha_{j k} q_{k}+\sum_{k=1}^{6} \beta_{j k} \dot{q}_{k} .
$$

By combining Eqs. (23)-(26) together, the equations of motion can be written in a compact matrix form as

$$
\mathrm{M} \ddot{Q}=\mathrm{S}\left(\begin{array}{l}
Q \\
\dot{Q}
\end{array}\right),
$$

with the obvious notation $\ddot{Q}=\left\{\ddot{q}_{1}, \ldots, \ddot{q}_{6}\right\}$. In Eq. (27), $\mathbb{M}$ is the $n \times n(n=6)$ "mass-matrix" composed by the $b_{j k}$ coefficients

$$
\mathbb{M}_{j k}=2 b_{j k} \delta_{j k}+b_{j k}\left(1-\delta_{j k}\right),
$$

where the factor of 2 on the diagonal elements is a consequence of the restricted $j<k$ sum in the expansion (25). S is a $n \times 2 n$ matrix containing the $a_{j k}, c_{j k}, \alpha_{j k}$, and $\beta_{j k}$ coefficients,

$$
\mathrm{S}=\mathrm{A}_{2}+\mathrm{C}_{2}+\mathrm{A}_{1}+\mathrm{B}_{1},
$$

with

$$
\begin{aligned}
\mathrm{A}_{2 j k} & =\mathrm{A}_{2 k j}=2 a_{j k} \delta_{j k}+a_{j k}\left(1-\delta_{j k}\right), \quad k \leqslant n \\
& =0, \quad n<k \leqslant 2 n, \\
\mathrm{C}_{2 j k} & =0, \quad n \leqslant n \\
& =-\mathrm{C}_{2 k j}=c_{j k}-c_{k j}, \quad n<k \leqslant 2 n, \\
\mathrm{~A}_{1 j k} & =\mathrm{A}_{1 k j}=\alpha_{j k}, \quad k \leqslant n
\end{aligned}
$$

$$
=0, \quad n<k \leqslant 2 n,
$$

and

$$
\begin{aligned}
\mathbb{B}_{1 j k} & =0, & k \leqslant n \\
& =\mathbb{B}_{1 k j}=\beta_{j k}, & n<k \leqslant 2 n .
\end{aligned}
$$

Note that while $\mathbb{M}$ and the submatrix defined by the first $n$ $=6$ columns of $A_{2}$ are symmetric, the submatrix defined by the second $n=6$ columns of $\mathrm{C}_{2}$ is antisymmetric, as expected after inspection of the expansion (23).

For all practical purposes, it is convenient to turn (27) into a more symmetric form involving only first-order time derivatives. To this aim, we define the $2 n=12$-component vector $X$ as

$$
\begin{aligned}
& X_{2 j-1}=q_{j}, \quad j=1, \ldots, n=6 . \\
& X_{2 j}=\dot{q}_{j}, \quad
\end{aligned}
$$
tain

By inserting the definition (34) into (27), we finally ob-

$$
\dot{X}=A X,
$$

where $A$ is now the square $2 n \times 2 n$ dynamical matrix defined from $\mathrm{M}^{-1}$ and $\mathrm{S}$ after inserting rows of zeros.

$$
A_{j k} \begin{cases}\left(\mathbb{M}^{-1} \mathrm{~S}\right)_{j 1+k-1 / 2}, & j \text { even and } k \text { odd } \\ \left(\mathbb{M}^{-1} \mathrm{~S}\right)_{j 7+k-2 / 2}, & j \text { even and } k \text { even } \\ 1, & j \text { odd and } k=j+1 \\ 0, & j \text { odd and } k \neq j+1 .\end{cases}
$$

The relations (35) and (36) are central equations, written in a form amenable for numerical evaluation. The eigenvalues of the $A$ matrix (36) correspond to the normal modes of the rotor and the solution of the set of differential equations (35) completely determines the small-amplitude dynamical behavior of the rotor modeled in Fig. 3. Before turning to the description of the numerical method, we introduce rotating and nonrotating dampings.

\section{Rotating and nonrotating dampings}

By means of (25) and (26) we can in principle introduce any known force determining the dynamical behavior of the rotor. In the following we include dissipative forces $\mathcal{R}_{R}$ and $\mathcal{R}_{\mathrm{NR}}$ due to rotating and nonrotating damping mechanisms respectively, (see Refs. 19 and 20). The rotating part of the dissipative force is to be ascribed to dissipation of the laminar suspensions. In supercritical rotation, this kind of dissipation is known to destabilize the system, generating whirl motions. It can be expressed as

$$
\begin{aligned}
\mathcal{R}_{R}(Q, \dot{Q})= & -\Gamma_{R a}\left[\dot{\hat{L}}_{a}-\left(\dot{\hat{L}}_{a} \cdot \hat{Z}^{\prime}\right) \cdot \hat{Z}^{\prime}\right] \\
& -\sum_{\lambda=o, i} \Gamma_{R \lambda}\left[\dot{\hat{L}}_{\lambda}-\left(\dot{\hat{L}}_{\lambda} \cdot \hat{R}_{a}(Q)\right]\left[\hat{R}_{a}(Q)\right],\right.
\end{aligned}
$$

where the velocities $\dot{\hat{L}}_{\lambda}$ are functions of $(Q, \dot{Q})$. Instead, nonrotating damping has the effect of stabilizing a system in supercritical rotation and can be written as

$$
\mathcal{R}_{\mathrm{NR}}(Q, \dot{Q})=-\Gamma_{\mathrm{NR}}\left[\mathbf{V}_{i}(Q, \dot{Q})+\boldsymbol{\omega}_{s} \times \mathbf{R}_{i}(Q)\right]
$$


Other forces acting on the rotor, such as external disturbances due-for instance-to tides and seismic noise, or control forces applied in order to control the rotor dynamics, can also be included, as described in Sec. IV.

\section{THE NUMERICAL METHOD}

\section{A. General considerations}

The simulation method that we have implemented rigorously follows the derivation outlined in Sec. III. We have found very convenient to use the MATLAB environment, with SYMBOLIC TOOLBOX and SIMULINK packages, as it allows us to perform all the needed symbolic calculations and numerical evaluations, together with the analysis of experimental data.

We start from the formal Lagrange function written in a user-friendly way as in (3)-(7) and (10)-(12) by means of symbolic vector operations. We specify the choice (13) for the generalized coordinates with respect to the $\left\{X^{\prime} Y^{\prime} Z^{\prime}\right\}$ reference frame and define accordingly all the vectors entering $\mathcal{L}$. We then move on to the symbolic computation by linearizing and expanding the Lagrange function as in (23) and define the matrices $\mathbb{M}, A_{2}, C_{2}, A_{1}, B_{1}$, and $A$.

Once the system parameters are fixed (see below), the numerical computation is carried out using standard packages to find eigenvalues and eigenvectors of the $A$ matrix, which are the normal frequencies and modes of the spinning rotor. The $A$ matrix is then inserted as input to perform the dynamical simulation within standard transfer-function method used in the SIMULINK toolbox.

The advantage of this strategy is apparent, in that it easily allows us to make any changes in the model that correspond to changes in the experiment we would like to test before implementation. Since the number of bodies $n_{b}$ and of the generalized coordinates $n$ are symbolically defined and specified only once, all what is to be done in order to introduce any changes or new features amounts to modification or addition of pieces of the Lagrangian after having symbolically written them in terms of vector operations.

The description of the method used to introduce external forces is postponed to Part II of this work, where it is used to evaluate the common mode rejection function. We now turn to listing the system parameters.

\section{B. System parameters}

The parameters which govern the physics of the GGG rotor are the geometrical dimensions of the three bodies, their weight, the mounting error $\epsilon$, the elastic constants, length and anisotropy factor $\Lambda$ of the three laminar suspensions, and the quality factor $\mathcal{Q}$. To these parameters-which are fixed after construction-we must add the spin frequency $\nu_{s}=\omega_{s} / 2 \pi$ that can be varied in the course of the experiment. The balancing of the beams and the natural period $T_{D}$ of oscillation of the test cylinders relative to one another can also be adjusted, as discussed earlier by moving small masses along the balance (coupling) arm.

We have inserted as inputs to the numerical calculation all the above parameters as determined in the real GGG instrument. They are listed in Tables I and II. As for the spin
TABLE I. Input parameters for the numerical calculations: geometrical dimensions of the real bodies. (A mounting error of $\epsilon=20 \mu \mathrm{m}$ has also been used.)

\begin{tabular}{|c|c|c|c|c|c|}
\hline Body & $\begin{array}{c}m_{\lambda} \\
(\mathrm{kg})\end{array}$ & $\begin{array}{l}R_{\lambda I} \\
(\mathrm{~cm})\end{array}$ & $\begin{array}{l}R_{\lambda E} \\
(\mathrm{~cm})\end{array}$ & $\begin{array}{c}L_{\lambda} \\
(\mathrm{cm})\end{array}$ & $\begin{array}{l}R_{\lambda H} \\
(\mathrm{~cm})\end{array}$ \\
\hline $\operatorname{Arm}(\mathrm{a})$ & 0.3 & 3.3 & 3.5 & $\begin{array}{c}19 \\
(\Delta L=-0.1353)\end{array}$ & $2 L_{a}+\Delta L$ \\
\hline Outer cyl. (o) & 10 & 12.1 & 13.1 & $2 L_{a}+L_{i}+\Delta L$ & 29.8 \\
\hline Inner cyl. (i) & 10 & 8.0 & 10.9 & 4.5 & 21.0 \\
\hline
\end{tabular}

frequency, in the experiment it varies in the range $\left(0 \leqslant \nu_{s}\right.$ $\leqslant 3.9) \mathrm{Hz}$, while in the model calculations it can be assumed in a wider range $\left(0 \leqslant \nu_{s} \leqslant 10\right) \mathrm{Hz}$.

The differential periods $T_{D}$ corresponding to the value $\Delta L$ listed in Table I are measured to be 11.7 and $10.8 \mathrm{~s}$ in the $X$ and $Y$ directions, respectively. These values are in reasonable agreement with the following simple formula:

$$
T_{D}=\frac{2 \pi}{\sqrt{\left(K+K_{i}+K_{o}\right) l^{2} /\left(m_{i}+m_{o}\right) L_{a}^{2}-\left(g / 2 L_{a}\right)\left(\Delta L / L_{a}\right)}},
$$

which can be derived from the general equations of motion (24) describing the small oscillations of the $\theta_{\lambda}$ angles, in the very simplified case in which the bodies are neither rotating nor subjected to any dissipative or other external forces, except gravity, and under the reasonable assumption that $\theta_{i}$ $=\theta_{o}=0$ and that $\phi_{\lambda}$ 's are constant, e.g., $\phi_{\lambda}=0$.

Of the whole set of parameters used, only the anisotropy factor $\Lambda$ of the suspensions and the construction and mounting error $\epsilon$ are not measured from the instrument. $\Lambda$ is tuned, together with the balancing $\Delta L$, so as to reproduce the natural frequencies of the nonspinning instrument. A conservative value $\epsilon=20 \mu \mathrm{m}$ is assumed for the offset, and it is checked a posteriori not to have any sizable effect on these results.

\section{RESULTS: THE NORMAL MODES}

We have solved for the eigenvalues $\nu_{n}$ of the matrix $A$ in (35), using the system parameters listed in Sec. IV B.

Figure 4 summarizes our results by plotting the normal modes of the system ( $\nu_{n}$ in the nonrotating frame) as functions of the spin frequency $\nu_{s}=\omega_{s} / 2 \pi$ of the rotor. In this figure, theoretical results for $\nu_{n}\left(\nu_{s}\right)$ 's are displayed by the solid lines in the case of zero rotating damping (i.e., no dissipation in the suspensions) and by the open circles in the case of nonzero rotating damping $\left[\mathcal{Q}\left(\nu_{s}\right)=510\right]$. At zero damping there are 12 lines, 6 horizontal and 6 inclined; start-

TABLE II. Input parameters for the numerical calculations: laminar suspensions data. In addition, a conservative value $\mathcal{Q}\left(\nu_{s}\right)=510$ has been used taken from previous measurements of whirl growth (Ref. 14, Fig. 7).

\begin{tabular}{lccc}
\hline \hline Suspension & $l$ & $K$ & $\begin{array}{c}\text { Anisotropy } \\
(\mathrm{cm})\end{array}$ \\
\hline Central & 0.5 & $10^{6}\left(l / L_{Y^{\prime}} / K_{X^{\prime}}\right)$ & 2.6 \\
Outer cyl. (o) & 0.5 & $10^{6}$ & 1.0 \\
Inner cyl. (i) & 0.5 & $10^{6}$ & 1.0 \\
\hline \hline
\end{tabular}




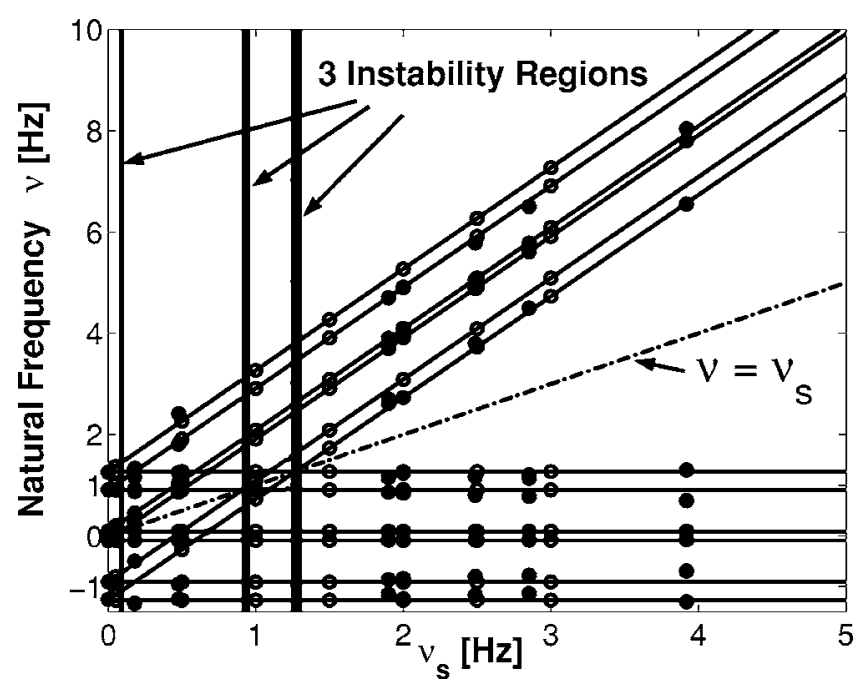

FIG. 4. Normal modes of the GGG rotor: the frequencies of the normal modes are plotted as functions of the spin frequency $\nu_{s}$. The normal modes as predicted theoretically assuming anisotropic suspensions are shown as 12 solid lines in the case of zero rotating damping and as open circles in the case of nonzero damping (see text). The experimental results are plotted as filled circles and clearly agree with the theoretical predictions. The bisecting dot-dashed line $\nu=\nu_{s}$ separates the supercritical $\left(\nu_{s}>\nu\right)$ from the subcritical $\left(\nu_{s}<\nu\right)$ region. Three vertical thick lines are plotted in correspondence of three instability regions, their thickness referring to the width of the regions (see Sec. V G).

ing from the three natural frequencies (Sec. II C 2) of the system, we get $3 \times 2 \times 2=12$ normal mode lines, a factor of 2 being due to anisotropy of the suspensions in the two orthogonal directions of the plane, and the other to the positive and negative signs (i.e., counterclockwise or clockwise whirl motion). For the nonzero damping case (open circles) a conservative low value $\mathcal{Q}\left(\nu_{s}\right)=510$ has been assumed (see Table II), referring to a comparatively large dissipation. This value has been obtained from previous not so favorable measurements of whirl growth, while much higher $\mathcal{Q}$ values (namely, much smaller dissipations) are expected (see discussion on this issue in Ref. [15], Sec. III). In Fig. 4, to be compared with the above theoretical results, we plot, as filled circles, the experimental results too, finding an excellent agreement between theory and experiments. Since Fig. 4 contains the crucial results of this work, it is worth discussing its main features in detail. The main features are the comparison with the experiment, the role of damping, the behavior at low spin frequencies, the so-called scissors's shape, the splitting of the normal modes, and the presence of three instability regions.

\section{A. Comparison with the experiment}

In the experiment, the rotor is first accelerated to spin at a given frequency $\nu_{s}$. Then, the natural modes are excited by means of capacitance actuators (indicated as OP in Fig. 1) in the $X^{\prime}$ or $Y^{\prime}$ directions at frequencies close to the natural frequencies $\nu_{n}^{0} \equiv \nu_{n}\left(\nu_{s}=0\right)$ of the system at zero spin. The excitation is performed for several (typically ten) fundamental cycles $1 / \nu_{n}^{0}$ by means of voltages applied to four of the eight outer plates (OP in Fig. 1). The actuators are then switched off and the bodies's displacements are recorded as functions of time by means of the readout described in Sec.
II B. A standard data analysis is then performed by fitting the measurement data to extract oscillation frequencies and damping of the modes.

The experimental data, resulting from averaging over several measurements, are represented as filled circles in Fig. 4. The agreement between theory and experiments is excellent, thus validating the model developed in Sec. III.

In the experimental spectra as well as in the theory, it is found that the amplitudes of the modes in the subcritical region $\nu_{s}<\nu$-represented by the inclined lines, with their open and filled circles-are quite small, while the nondispersive modes (the horizontal lines, not varying with the spin frequency) are preferably excited. When the horizontal lines cross the inclined ones, the latter modes can also be excited. Since the excited modes must obviously be avoided in operating the experiment, this information is very useful, in that it is telling us that we should avoid to spin the system at frequencies where these line crossings occur. Even more so, spin frequencies lying in the instability regions must be avoided (see Sec. V G).

\section{B. Role of damping}

We have numerically checked that the dissipation present in the system does not significantly shift the natural mode frequencies. This is apparent in Fig. 4, where the results obtained with damping (open circles) stay on the solid lines obtained in the absence of damping. It is worth stressing that this result is especially good because, as discussed above, we have used a low value $\mathcal{Q}\left(\nu_{s}\right)=510$, corresponding to comparatively large dissipations. As expected, the dissipation affects the line shape of the peaks, making them wider than in the absence of damping.

\section{Low-frequency limit}

At zero spin frequency we have recovered the theoretical and experimental results previously obtained for the nonrotating system. On the left hand side of Fig. 5, a zoom from Fig. 4 at very low spin frequencies, we can see that the nonspinning rotor is characterized by three natural frequencies for the instrument with ideally isotropic springs, the three bodies oscillating in a vertical plane. The frequency $\nu^{0}=0.09 \mathrm{~Hz}$ corresponds to the differential mode, where the centers of mass of the two test bodies oscillate in opposition of phase; the frequencies $\nu^{0}=0.91 \mathrm{~Hz}$ and $\nu^{0}=1.26 \mathrm{~Hz}$ correspond to common modes, in which the common center of mass of the two test bodies is displaced from the vertical. During rotation, the number of degrees of freedom increases to six, as discussed in Sec. III B, leading to the six lines plotted in the same figure.

We may get a flavor of the $\nu_{s}$ dependence of the modes in the $\nu_{s} \ll \nu_{n}$ limit, by evaluating the natural frequencies of only one spinning cylinder with mass $m$ and moments of inertia $I_{\xi}$ and $I_{\zeta}$. The cylinder is suspended at distance $L$ and with offset $\epsilon$ from a fixed frame by means of a cardanic suspension with isotropic elastic constant $K$ and length $l$. The calculation is performed by following the steps outlined in 

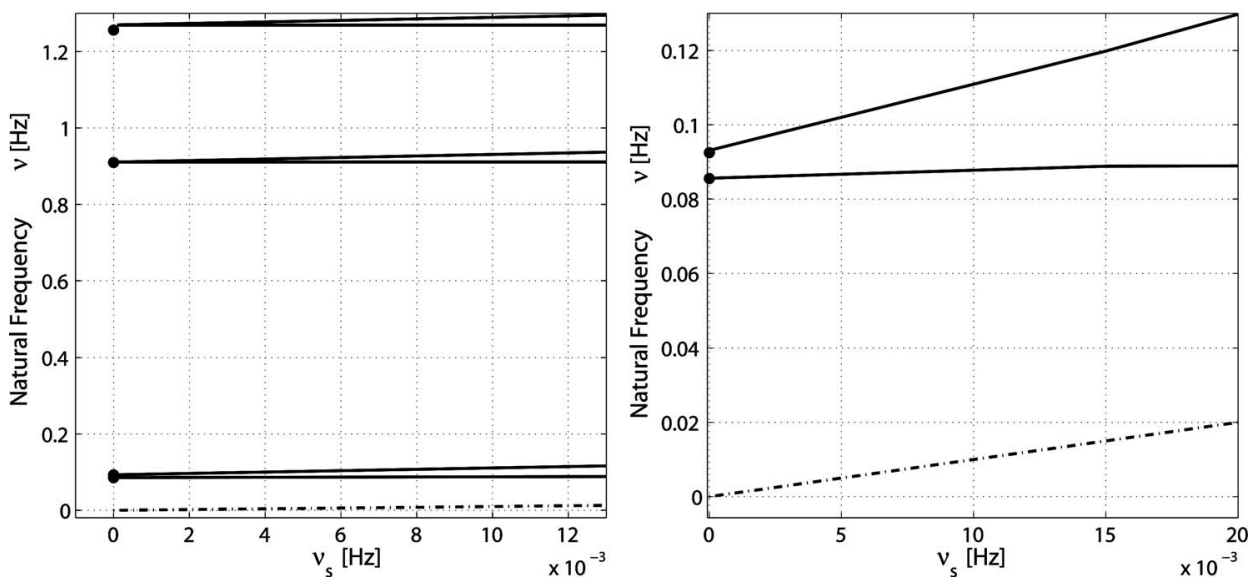

FIG. 5. Normal modes of the GGG rotor. On the left hand side we show a zoom from Fig. 4 in the very low spin frequency region, showing, in particular, the three-natural frequencies of the system in the zero spin case. On the right hand side, we plot a zoom from Fig. 4 in the small frequency region of both axes, showing the splitting into two lines of the low frequency mode because of anisotropy of the suspensions (the dashed line is the $\nu_{s}=\nu$ line as in Fig. 4).
Sec. III with $n_{b}=1$ and thus $n=4$. After evaluating the $A$ matrix [Appendix B, see Eq. (B10)] and solving $\operatorname{det}(A-s I)$ $=0$, we obtain the two double solutions

$$
\nu_{n 1,2,3,4}= \pm \widetilde{\nu}_{n}^{0} \frac{L}{L^{\prime}},
$$

for the four $\nu_{n}\left(\nu_{s} / \nu_{n} \rightarrow 0\right)$ in the nonrotating frame. In Eq. (40), $\widetilde{\nu}_{n}^{0}=(2 \pi)^{-1} \sqrt{g / L+K l^{2} / m L^{2}}$ is the natural frequency for the nonspinning pointlike mass. $L^{\prime}=\sqrt{L^{2}+\left(I_{\xi}-2 I_{\zeta}\right) / m}$ (with $I_{\zeta}<0.5 m L^{2}+I_{\xi}$, see Appendix B) takes into account the extended nature of the body and the ratio $L / L^{\prime}$ modifies $\nu_{n}$ with respect to $\widetilde{\nu}_{n}^{0}$.

\section{Anisotropy}

If the suspensions are not isotropic in the two orthogonal directions, as it is indeed the case for our real cardanic suspensions, each natural frequency is expected to split up. This is clearly shown on the right hand side of Fig. 5. It is worth noting that the splitting is larger for the lowest-frequency mode.

\section{E. Scissors's shape}

Figure 4 shows that each natural frequency of the nonspinning system splits up into two branches at $\nu_{s}>0$, a lower branch remaining approximately constant and an upper branch increasing with $2 \nu_{s}$.

This characteristic scissors's shape can be traced back to the general properties of spinning bodies (see also Part II). We again use the one-cylinder simple case (see Appendix B) to prove this statement. By following the same procedure which has led to Eq. (40) we obtain [see Eq. (B9)]

$$
\begin{aligned}
& \nu_{1,2}= \pm \sqrt{\nu_{s}^{2}-2 \nu_{n} \nu_{s} \frac{L}{L^{\prime}}} \simeq \pm \nu_{s}\left(1-\frac{\nu_{n}}{\nu_{s}} \frac{L}{L^{\prime}}\right), \\
& \nu_{3,4}= \pm \sqrt{\nu_{s}^{2}+2 \nu_{n} \nu_{s} \frac{L}{L^{\prime}}} \simeq \pm \nu_{s}\left(1+\frac{\nu_{n}}{\nu_{s}} \frac{L}{L^{\prime}}\right),
\end{aligned}
$$

showing that in the rotating frame $\nu_{n} \propto \nu_{s}$ to zeroth order. After taking the $\nu_{s} / \nu_{n} \rightarrow \infty$ limit and tranforming back to the nonrotating frame by means of the substitution $s_{n}=2 \pi i \nu_{n}$ $\rightarrow 2 \pi\left(i \nu_{n}+i \nu_{s}\right)$, we finally have

$$
\begin{aligned}
& \nu_{1,3} \simeq \pm \nu_{n} \frac{L}{L^{\prime}}, \\
& \nu_{2,4} \simeq 2 \nu_{s} .
\end{aligned}
$$

\section{F. Mode splitting}

The two branches may cross at selected frequencies. Crossing and anticrossing of degenerate modes are a very general concept, which applies to a variety of physical systems, from classical to quantum mechanics, from single to many-particle physics. As it is well known, ${ }^{24}$ splitting of the modes is expected in correspondence of such crossings. In our numerical results we have found all the 15 splittings expected for our system (see Fig. 4). Figure 6 shows a particular case of anticrossing of two modes.

\section{G. Instability regions}

Dynamical instability may occur whenever the values of the natural frequencies are in proximity of the spin frequency. In such regions the oscillation amplitude grows exponentially.

This is a well-known characteristic of rotating machines; in engineering books it is usually described within the simple model of the so-called Jeffcott rotor. ${ }^{21}$ The number of instability regions can be predicted from Fig. 4 after drawing the dotted-dashed line $\nu=\nu_{s}$. We have found indeed three insta-

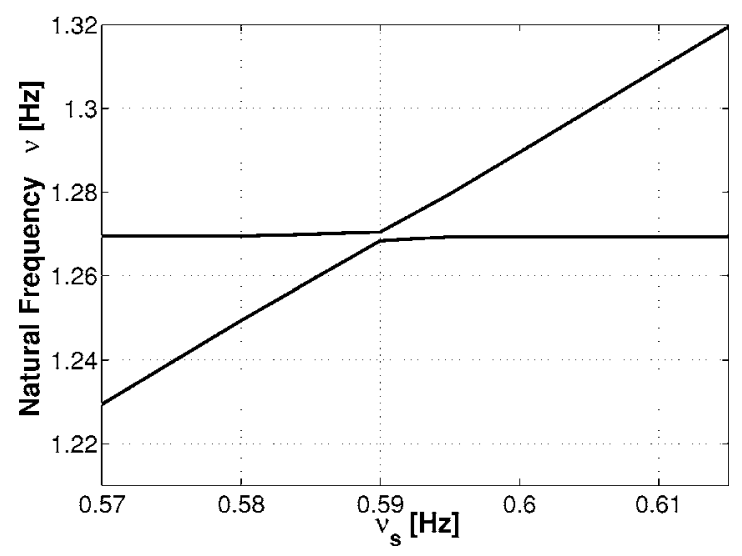

FIG. 6. Normal modes of the GGG rotor. A zoom from Fig. 4 showing one particular case of anticrossing of two modes. 


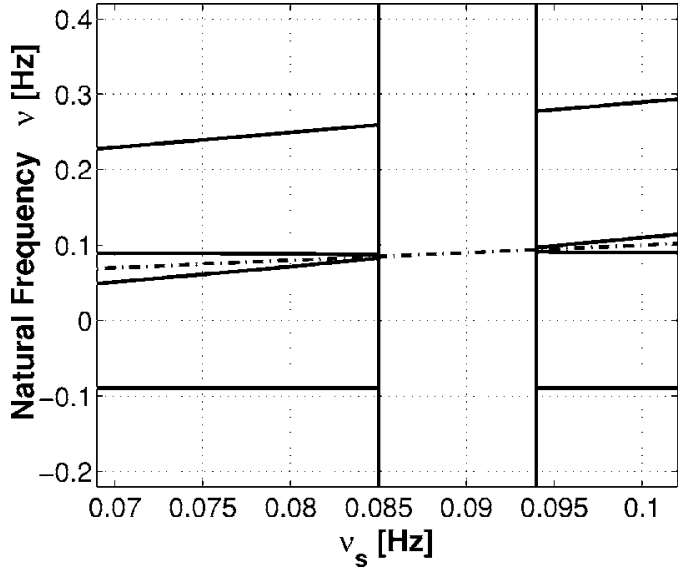

FIG. 7. Normal modes of the GGG rotor. The lowest-frequency instability region is zoomed in from Fig. 4.

bility regions. Figure 7 displays in detail the one at the lowest frequency; as shown in Fig. 4, the two at higher frequencies are found to be wider and closer to each other. These theoretical results do explain why in the experiment we can increase the spin frequency and cross the low-frequency instability region easily, while it is much more difficult to cross the frequency range $0.9 \rightarrow 1.3 \mathrm{~Hz}$. In the past we solved this problem by designing and installing passive dampers to be switched on from remote just before resonance crossing, and then turned off at higher spin frequencies; the least noisy was a special, no oil damper described in Ref. 25, p. 45. Later on the GGG rotor imperfections have been reduced so that all instability regions can now be crossed, if the crossing is sufficiently fast, without producing any relevant disturbances even in absence of a passive damper. The physical space previously occupied in the vacuum chamber by the passive damper is now used for the inductive power coupler, indicated as PC in Fig. 1, which provides the necessary power to the rotating electronics and has allowed us to avoid noisy sliding contacts.

\section{CONCLUDING REMARKS}

We have demonstrated that the linearized model set up in Sec. III can quantitatively account for the dynamical response of the GGG rotor, an apparatus designed to test the equivalence principle with fast rotating, weakly coupled, macroscopic, concentric cylinders (Sec. II). The model developed here can be expanded to include external disturbances whose effects need to be taken into account in testing the equivalence principle. A qualitative understanding has been provided, by means of helpful analytical solutions of the simplified model under special limits, of relevant features observed in the simulations as well as in the experimental data.

We have acquired a detailed knowledge of the instrument's features and the way it works, the main feature being the normal modes of the system (Sec. V) in the whole range of spin frequencies, from subcritical to supercritical regime, and as functions of the governing parameters (see Sec. IV B).

In particular, we have established the location and characteristics of the instability regions; we have verified quan- titatively the effects of dissipation in the system, showing that losses can be dealt with and are not a matter of concern for the experiment; we have established the split up of the normal modes into two scissorlike branches, distinguishing modes which are preferentially excited (the horizontal lines) from those whose spectral amplitudes are typically small (the inclined lines), thus learning how to avoid the spin frequencies corresponding to their crossings, in order not to excite the quiet modes too by exchange of energy; we have investigated the self-centering characteristic of the GGG rotor when in the supercritical rotation regime, gaining insight on how to exploit this very important physical property for improving the quality of the rotor, hence its sensitivity as a differential accelerometer.

In the following Part II of this work we apply the same model and methods developed here to investigate the common mode rejection behavior of the GGG rotor, a crucial feature of this instrument devoted to detect extremely small differential effects.

\section{ACKNOWLEDGMENT}

Thanks are due to INFN for funding the GGG experiment in its Laboratory of San Piero a Grado in Pisa.

\section{APPENDIX A: THE LAGRANGE FUNCTION IN THE ROTATING REFERENCE FRAME}

In the following, in order to simplify the notation we drop the $\lambda$ indices everywhere and restrict our reasoning to only one body. Let us begin with the expression (4). After using Eq. (8) into (4), we have

$$
\mathcal{T}=\frac{1}{2} \int_{\tau_{i}}\left[\mathbf{V}^{2}+(\boldsymbol{\Omega} \times \boldsymbol{\rho})^{2}+2 \mathbf{V} \cdot(\boldsymbol{\Omega} \times \boldsymbol{\rho})\right] d m .
$$

We conveniently represent the vectors $\boldsymbol{\Omega}=\overleftrightarrow{\mathcal{M}} \boldsymbol{\Omega}_{\xi \eta \zeta}$ and $\boldsymbol{\rho}=\overleftrightarrow{\mathcal{M}} \boldsymbol{\rho}_{\xi \eta \zeta}$ in the $\{\xi \eta \zeta\}$ frame by means of the rotation matrix

$$
\overleftrightarrow{\mathcal{M}}=\left(\begin{array}{lll}
\sin \phi & \cos \theta \cos \phi & -\sin \theta \cos \phi \\
-\cos \phi & \cos \theta \sin \phi & -\sin \theta \sin \phi \\
0 & \sin \theta & \cos \theta
\end{array}\right),
$$

with respect to the $\left\{X^{\prime} Y^{\prime} Z^{\prime}\right\}$ reference system.

Thus, by exploiting the properties of the vectorial product and the definition of center of mass, namely, $\int_{\tau_{i}} \rho_{\xi}$ $=\int_{\tau_{i}} \rho_{\eta}=\int_{\tau_{i}} \rho_{\zeta}=0$, we find the following results for the integrals appearing in Eq. (A1):

$$
\begin{aligned}
& \frac{1}{2} \int_{\tau_{i}} \mathbf{V}^{2} d m=\frac{1}{2} m \mathbf{V}^{2}, \\
& \frac{1}{2} \int_{\tau_{i}}(\boldsymbol{\Omega} \times \boldsymbol{\rho})^{2} d m=\frac{1}{2} \sum_{\alpha} I_{\alpha \alpha} \Omega_{\alpha}^{2}, \\
& \frac{1}{2} \sum_{\alpha} I_{\alpha \alpha} \Omega_{\alpha}^{2}=\frac{1}{2} I_{\dot{\xi}}\left(\dot{\phi}^{2} \sin ^{2} \theta+\dot{\theta}^{2}\right),
\end{aligned}
$$




$$
\begin{aligned}
& \int_{\tau_{i}} \mathbf{V} \cdot(\boldsymbol{\Omega} \times \boldsymbol{\rho}) d m=(\mathbf{V} \times \boldsymbol{\Omega}) \cdot \int_{\tau_{i}} \boldsymbol{\rho} d m=0, \\
& \int_{\tau_{i}} \mathbf{V} \cdot\left(\boldsymbol{\omega}_{s} \times \mathbf{R}\right) d m=m \mathbf{V} \cdot\left(\boldsymbol{\omega}_{s} \times \mathbf{R}\right), \\
& \int_{\tau_{i}} \mathbf{V} \cdot\left(\boldsymbol{\omega}_{s} \times \boldsymbol{\rho}\right) d m=\left(\mathbf{V} \times \boldsymbol{\omega}_{s}\right) \cdot \int_{\tau_{i}} \boldsymbol{\rho} d m=0, \\
& \int_{\tau_{i}}(\boldsymbol{\Omega} \times \boldsymbol{\rho}) \cdot\left(\boldsymbol{\omega}_{s} \times \mathbf{R}\right) d m=\left(\boldsymbol{\omega}_{s} \times \mathbf{R}\right) \cdot \int_{\tau_{i}}(\boldsymbol{\Omega} \times \boldsymbol{\rho})=0, \\
& \int_{\tau_{i}}(\boldsymbol{\Omega} \times \boldsymbol{\rho}) \cdot\left(\boldsymbol{\omega}_{s} \times \boldsymbol{\rho}\right) d m=I_{\xi} \boldsymbol{\omega}_{s} \dot{\boldsymbol{\phi}} \sin ^{2} \theta+I_{\zeta} \omega_{s}^{2} \cos \theta, \\
& \frac{1}{2} \int_{\tau_{i}}\left(\boldsymbol{\omega}_{s} \times \mathbf{R}\right)^{2} d m=\frac{1}{2} m\left(\boldsymbol{\omega}_{s} \times \mathbf{R}\right)^{2}, \\
& \frac{1}{2} \int_{\tau_{i}}\left(\boldsymbol{\omega}_{s} \times \boldsymbol{\rho}\right)^{2} d m=\frac{1}{2}\left[I_{\xi} \sin ^{2} \theta+I_{\zeta} \cos ^{2} \theta\right] \omega_{s}^{2}, \\
& \int_{\tau_{i}}\left(\boldsymbol{\omega}_{s} \times \mathbf{R}\right) \cdot\left(\boldsymbol{\omega}_{s} \times \boldsymbol{\rho}\right) d m=\left(\boldsymbol{\omega}_{s} \times \mathbf{R}\right) \cdot \int_{\tau_{i}}\left(\boldsymbol{\omega}_{s} \times \boldsymbol{\rho}\right) d m \\
& =0 .
\end{aligned}
$$

By collecting all these results, one ends up with the final forms (17)-(19) for the original $\mathcal{T}$ function (4).

\section{APPENDIX B: THE ONE-CYLINDER SOLUTION}

It is useful to study (along the lines of Sec. III) the simplified case of only one spinning cylinder with mass $m$ and moments of inertia $I_{\xi}$ and $I_{\zeta}$. This amounts to setting $n_{b}=1$ and thus $n=4$ for the number of generalized coordinates.

The $A$ matrix turns out to be

$$
A=\left(\begin{array}{llll}
0 & 1 & 0 & 0 \\
\mathcal{L}_{11} / \mathcal{L}_{22} & \mathcal{R}_{12} / \mathcal{L}_{22} & \mathcal{R}_{13} / \mathcal{L}_{22} & \left(\mathcal{L}_{14}-\mathcal{L}_{23}\right) / \mathcal{L}_{22} \\
0 & 0 & 0 & 1 \\
\mathcal{Q}_{31} / \mathcal{L}_{44} & \left(\mathcal{L}_{23}-\mathcal{L}_{14}\right) / \mathcal{L}_{44} & \mathcal{L}_{33} / \mathcal{L}_{44} & \mathcal{R}_{34} / \mathcal{L}_{44}
\end{array}\right),
$$

where the coefficients of $\mathcal{L}$ and $\mathcal{R}$ are defined in terms of the system parameters and of the equilibrium positions $\theta_{0}$ and $\phi_{0}$ as

$$
\begin{aligned}
\mathcal{L}_{11}= & m \omega_{s}^{2} L\left(L \cos 2 \theta_{0}+\epsilon \sin \theta_{0}\right)-m g L \cos \theta_{0} \\
& -K l^{2} \cos 2 \theta_{0}+\left(I_{\xi}-I_{\xi}\right) \omega_{s}^{2} \cos 2 \theta_{0}-I_{\zeta} \omega_{s}^{2} \cos \theta_{0}, \\
\mathcal{L}_{22}= & m L^{2}+I_{\xi}, \\
\mathcal{L}_{33}= & m \omega_{s}^{2} \epsilon L \sin \theta_{0}, \\
\mathcal{L}_{44}= & \left(m L^{2}+I_{\xi}\right) \sin ^{2} \theta_{0},
\end{aligned}
$$

$$
\begin{aligned}
\mathcal{L}_{14}=\mathcal{L}_{41}= & I_{\zeta} \omega_{s} \sin 2 \theta_{0}-m \omega_{s} \epsilon L \cos \theta_{0} \\
& +m \omega_{s}^{2} L^{2} \sin 2 \theta_{0}, \\
\mathcal{L}_{23}=\mathcal{L}_{32}= & -m \omega_{s} \epsilon L \cos \theta_{0},
\end{aligned}
$$

and

$$
\begin{aligned}
& \mathcal{R}_{12}=-\left(\Gamma_{R}+\Gamma_{\mathrm{NR}}\right) L^{2}, \\
& \mathcal{R}_{13}=-\Gamma_{\mathrm{NR}} \omega_{s} L \epsilon \cos \theta_{0}, \\
& \mathcal{R}_{31}=-\Gamma_{\mathrm{NR}} \omega_{s} L\left(L \sin 2 \theta_{0}+\epsilon\right) \\
& \mathcal{R}_{34}=-\left(\Gamma_{R}+\Gamma_{\mathrm{NR}}\right) L^{2} \sin ^{2} \theta_{0} .
\end{aligned}
$$

In the case of negligible dissipation, the eigenvalue equation $\operatorname{det}(A-s I)$ for $s=2 \pi i \nu$ reads

$$
s^{4}-s^{2}\left(a_{21}+a_{43}+a_{24} a_{42}\right)+a_{21} a_{43}=0 .
$$

In the $\omega_{s} \gg \omega_{n}$ limit, the equilibrium solutions are

$$
\theta_{0} \simeq \frac{\epsilon L}{L^{\prime 2}}\left[1+\left(\frac{L}{L^{\prime}}\right)^{2}\left(\frac{\widetilde{\omega}_{n}^{0}}{\omega_{s}}\right)^{2}\right], \quad \phi_{0}=0,
$$

where $L^{\prime}=\sqrt{L^{2}+\left(I_{\xi}-2 I_{\zeta}\right) / m}, I_{\zeta}<0.5 m L^{2}+I_{\xi}$, and

$$
\widetilde{\omega}_{n}^{0}=\sqrt{\frac{g}{L}+\frac{K l^{2}}{m L^{2}}}
$$

is the natural frequency of the pointlike mass. Equation (B6) becomes then

$$
s^{4}+2 s^{2} \omega_{s}^{2}\left[1+\left(\frac{\omega_{n}^{0}}{\omega_{s}}\right)^{2}\right]+\omega_{s}^{4}\left[1-2\left(\frac{\omega_{n}^{0}}{\omega_{s}}\right)^{2}\right]=0,
$$

where we have defined the natural frequency of the cylinder mass as $\omega_{n}^{0} \equiv\left(L / L^{\prime}\right) \widetilde{\omega}_{n}^{0}$.

In the $\omega_{s} \ll \omega_{n}$ limit, the eigenvalue equation becomes instead

$$
s^{4}+2 s^{2}\left(\frac{L}{L^{\prime}}\right)^{2} \widetilde{\omega}_{n}^{02}+\left(\frac{L}{L^{\prime}}\right)^{4} \widetilde{\omega}_{n}^{04}=0 .
$$

Equations (B9) and (B10) are used to derive the results (40)-(44) in the main text.

\section{APPENDIX C: THE SELF-CENTERING}

This appendix is devoted to a key feature of the GGG experiment, namely, the concept of self-centering of the rotor in supercritical rotation. Let us analyze the one-cylinder case, by numerically integrating the equations of motion in the presence of nonrotating damping, to make the rotor asymptotically stable (Sec. III D 1). Figure 8 shows the resulting motion of the cylinder in the horizontal plane of the rotating reference frame: its center-of-mass spirals inward towards an equilibrium position much closer to the origin, i.e., to the rotation axis. The equilibrium position always lies in the same direction as the initial offset vector $\boldsymbol{\epsilon}$, which in this simulation was assumed to be in the $X^{\prime}$ direction. The center of mass of the cylinder will eventually perform smallamplitude oscillations around the asymptotic value $\left\{X_{0}^{\prime}\right.$ $\left.=\epsilon-L \sin \theta_{0}, Y_{0}^{\prime}=0\right\}$.

In the limit of small angles we obtain 


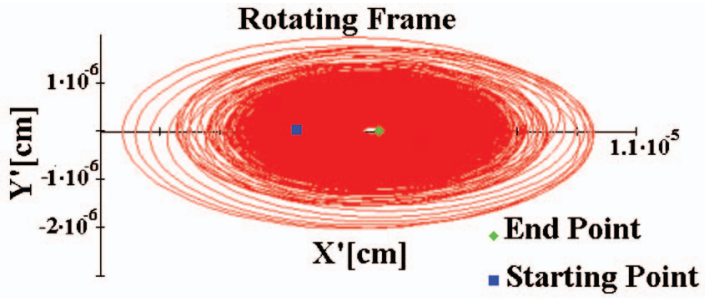

FIG. 8. (Color) Self-centering of one cylinder in the presence of nonrotating damping. Simulated $X^{\prime}(t)-Y^{\prime}(t)$ plot showing the motion of the center of mass of the cylinder in the rotating reference frame (one cylinder model, $\nu_{s}=5 \mathrm{~Hz}$ ). The center of mass spirals inward from the initial offset value and large initial oscillations to a final value, much closer to the rotation axis.

$$
\theta_{0} \simeq \pm \frac{\epsilon}{L}\left[\frac{1}{\left(L^{\prime} / L\right)^{2}-\left(\omega_{n} / \omega_{s}\right)^{2}}\right]
$$

with

$$
\phi_{0}=0(\pi),
$$

in the case of the lower (upper) sign in Eq. (C1), respectively (angles defined as in Fig. 3). The cylinder's center of mass is eventually located at a distance

$$
\Delta X \simeq \epsilon \pm L \theta_{0}=\epsilon-\left[\frac{\epsilon}{\left(L^{\prime} / L\right)^{2}-\left(\omega_{n} / \omega_{s}\right)^{2}}\right],
$$

from the rotation axis.

In Fig. 9 we plot, as function of the spin frequency $\nu_{s}$, the self-centering distance $\Delta X$ in the one-cylinder case discussed above and in the point masss case. According to the previous appendix, if $L^{\prime} \neq L$ we have the cylinder, while if $L=L^{\prime}$ we have the point mass. The two curves are worth comparing. They have a similar behavior till the resonance peak (in this case, at about $\nu_{s} \approx 1 \mathrm{~Hz}$ ); the distance from the rotation axis remains constant till, at spin frequencies slightly below the natural one, it starts increasing showing a typical peak at the resonance. For the cylinder and the point mass

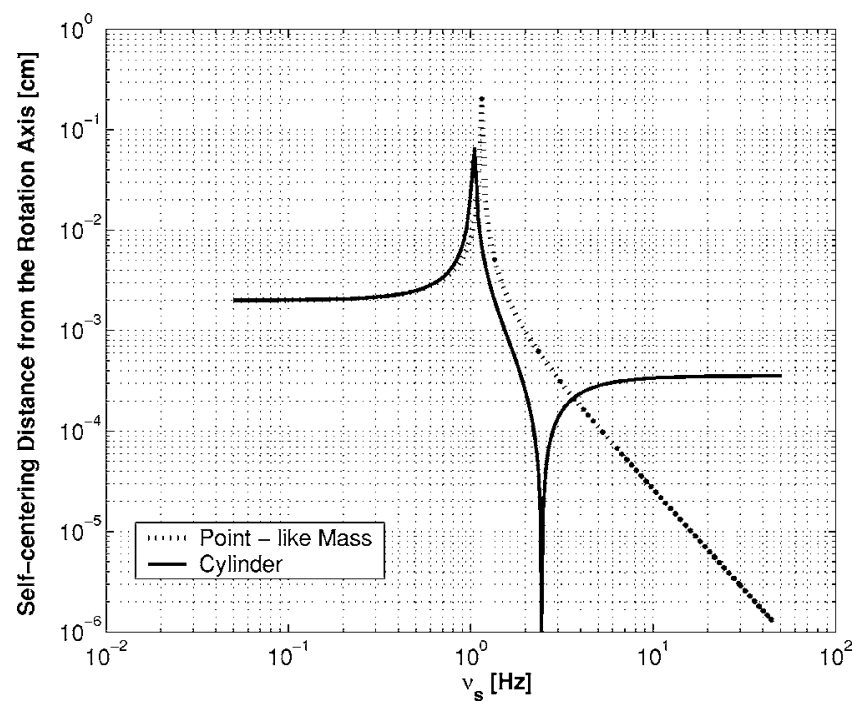

FIG. 9. Self-centering of one cylinder in the presence of nonrotating damping. The distance $\Delta X$ of the center of mass of the cylinder from the rotation axis is plotted as function of the spin frequency $\nu_{s}$, in agreement with Eq. (C3). The same distance in the case of a point mass is plotted as a dashed line. See text for comments on their comparison. the peaks are slightly shifted. The constant value can be obtained from Eq. (C3) in the limit of small spin frequencies $\omega_{s} \ll \omega_{n}$, finding

$$
\Delta X \simeq \epsilon .
$$

We can also recover the position and relative shift of the resonance peaks in the two cases from the values $\nu_{S}^{p}$ (the spin frequency at the peak) taken by the poles of $\Delta X$ in Eq. (C3), namely,

$$
\nu_{s}^{p}= \pm \frac{L}{L^{\prime}} \nu_{n} .
$$

Thus, the position of the peaks is dictated by the natural frequency $\nu_{n}$, while the shift is due to the difference between $L$ and $L^{\prime}$.

At rotation speeds above the resonance and in the highly supercritical regime $\omega_{s} \gg \omega_{n}$, the behavior of the cylinder and that of the point mass are remarkably different.

For the cylinder, $\Delta X$ drops to a minimum and then saturates at a constant value, while for the point mass it keeps decreasing monotonically. The minimum for the cylinder is related to the presence of a zero in Eq. (C3), namely,

$$
\nu_{s}^{z}= \pm \frac{L}{\sqrt{L^{\prime 2}-L^{2}}} \nu_{n}
$$

which is valid only if $L^{\prime}>L$. Instead, for the point mass we have the finite value $\Delta X=\epsilon /\left(1-\omega_{s}^{2} / \omega_{n}^{2}\right)$. Note that the position of the minimum shifts towards higher spin frequencies as $L^{\prime} \rightarrow L$, namely, as the finite cylinder case approaches a point mass. In the limit $\omega_{s} \gg \omega_{n}$ (i.e., highly supercritical speeds) Eq. (C3) yields

$$
\Delta X \simeq \epsilon\left[1-\left(\frac{L}{L^{\prime}}\right)^{2}\right],
$$

which explains the saturation to a constant self-centering value in the case of a finite cylinder, whereas a point mass would monotonically approach perfect centering (i.e., $\Delta X$ $=0)$.

In fact, it is very interesting to note that $\Delta X$ depends slightly on the point that we are considering along the cylinder's axis. In particular, in the limit $\omega_{s} \rightarrow \infty$, where $\Delta X$ of the cylinder's center of mass saturates, the point at distance $\tilde{L}$ $=L^{\prime 2} / L$ from the suspension point along the axis has instead perfect self-centering, namely, $\Delta X=0$. This is easily seen from Eq. (C3) after substituting $\epsilon \pm L \theta_{0}$ with $\epsilon \pm \tilde{L} \theta_{0}$ and imposing $\Delta X=0$. We plan to exploit this property in order to obtain better self-centering, though it needs further investigation in the actual GGG rotor.

We can consider a plot similar to that of Fig. 9 in the GGG case with two concentric cylinders and a coupling arm, where there are three natural frequencies (one differential and two common modes).

It happens that the common mode behavior is similar to that of the one-cylinder case (shown as a solid line in Fig. 9); namely, for each common mode frequency there is a resonance peak and a minimum peak. Instead, the differential frequency behavior is similar to that of a point mass (shown as a dashed line in Fig. 9). This latter fact is because in the 
differential mode the coupling arm oscillates and the cylinders's centers of mass move in the horizontal plane with the opposite phase, while $\theta_{i, o}=0$; under these conditions, their moment of inertia is irrelevant in determining the dynamics, which therefore is very much alike the case of a point mass. As a result, the $\Delta X$ of the GGG rotor for intermediate values of the spin frequency is characterized by one peak at low frequency, in correspondence to the differential mode, and two peaks and two minima, in correspondence to the common modes. Instead, in the limit of very low and very high spin frequencies, it has a behavior similar to that displayed in Fig. 9, depending on the values and directions assumed for the initial offsets of the three bodies.

Thus, in order to obtain the best possible centering of the test cylinders in the GGG rotor, one can either spin at a frequency close to the minima of the common modes, or above both of them, in such a condition that the two cylinders are better centered on their own rotation axes than both of them are, together, in a common mode. Self-centering on the rotation axes is very important in order to reduce rotation noise, because we are dealing with rapidly spinning macroscopic bodies and aiming at measuring extremely small effects. The issue therefore needs a careful investigation, and to this end realistic numerical simulations of the apparatus are an essential tool.

Finally, concerning the use of supercritical rotors for EP testing, it is worth mentioning a frequently asked question: Would a relative displacement of the test bodies caused by an external force-such as that resulting from an EP violation-be reduced by self-centering in supercritical rotation as it happens for the original offset $\epsilon$ ? The answer is "no" because the offset vector is fixed in the rotating frame of the system, while an external force gives rise to a displacement of the equilibrium position of the bodies in the nonrotating reference frame. In the presence of such a force, whirl motion will take place around the displaced position of equilibrium. A numerical simulation, showing this important feature is reported and discussed in Ref. 11, PLA paper, p. 176.

${ }^{1}$ R. V. Eötvös, D. Pekar, and E. Fekete, Ann. Phys. (N.Y.) 68, 11 (1922).

${ }^{2}$ P. G. Roll, R. Krotov, and R. H. Dicke, Ann. Phys. (N.Y.) 26, 442 (1964).
${ }^{3}$ V. B. Braginsky and V. I. Panov, Sov. Phys. JETP 34, 463 (1972).

${ }^{4}$ Y. Su et al., Phys. Rev. D 50, 3614 (1994).

${ }^{5}$ S. Baebler, B. R. Heckel, E. G. Adelberger, J. H. Gundlach, U. Schimidt, and H. E. Swanson, Phys. Rev. Lett. 83, 3585 (1999).

${ }^{6}$ T. Damour and A. M. Polyakov, Nucl. Phys. B 423, 532 (1994); Gen. Relativ. Gravit. 26, 1171 (1994).

${ }^{7}$ E. Fischbach, D. E. Krause, C. Talmadge, and D. Tadic, Phys. Rev. D 52, 5417 (1995).

${ }^{8}$ T. Damour, F. Piazza, and G. Veneziano, Phys. Rev. Lett. 89, 081601 (2002).

${ }^{9}$ P. W. Worden, Jr. and C. W. F. Everitt, in Experimental Gravitation, Proceedings of the "Enrico Fermi" International School of Physics, Course LVI, edited by B. Bertotti (Academic, New York, 1973); J. P. Blaser et al., ESA SCI Report No. (96)5, 1996 (unpublished); see also the STEP website http://einstein.stanford.edu/STEP/step2.html

${ }^{10}$ See the MICROSCOPE website http://www.onera.fr/dmph/accelerometre/ index.html

${ }^{11}$ A. M. Nobili, D. Bramanti, G. L. Comandi, R. Toncelli, E. Polacco, and M. L. Chiofalo, Phys. Lett. A 318, 172 (2003); "Galileo Galilei" (GG), Phase A Report, ASI (November 1998), 2nd ed. January 2000. A. M. Nobili, D. Bramanti, G. Comandi, R. Toncelli, E. Polacco, and G. Catastini, Phys. Rev. D 63, 101101 (2001); for a review see, e.g., Ref. 12; see also the GG website http://eotvos.dm.unipi.it/nobili

${ }^{12}$ A. M. Nobili, in Recent Advances in Metrology and Fundamental Constants, Proceedings of the "Enrico Fermi" International School of Physics, Course CXLVI, edited by T. J. Quinn, S. Leschiutta, and P. Tavella (IOS Press, 2001), p. 609.

${ }^{13} \mathrm{~W} . \mathrm{Li}$, http://linkage.rockfeller/edu/wli/1fnoise

${ }^{14}$ A. M. Nobili, D. Bramanti, G. L. Comandi, R. Toncelli, and E. Polacco, New Astron. 8, 371 (2003).

${ }^{15}$ G. L. Comandi, A. M. Nobili, D. Bramanti, R. Toncelli, E. Polacco, and M. L. Chiofalo, Phys. Lett. A 318, 213 (2003).

${ }^{16}$ A. M. Nobili, D. Bramanti, G. L. Comandi, R. Toncelli, E. Polacco, and M. L. Chiofalo, in Proceedings of the XXXVIIIth Rencontre de Moriond Gravitational Waves and Experimental Gravity, edited by J. Dumarchez and J. Tran Thanh Van (The Gioi, Vietnam, 2003), p. 371.

${ }^{17}$ G. L. Comandi, A. M. Nobili, R. Toncelli, and M. L. Chiofalo, Phys. Lett. A 318, 251 (2003).

${ }^{18}$ J. P. Den Hartog, Mechanical Vibrations (Dover, New York, 1985).

${ }^{19}$ S. H. Crandall, in Nonlinear Dynamics and Stochastic Mechanics, edited by W. Kliemann and N. S. Namachchivaya (CRC, Boca Raton, FL, 1995).

${ }^{20}$ G. Genta, Vibration of Structures and Machines (Springer-Verlag, New York, 1993).

${ }^{21}$ S. H. Crandall and A. M. Nobili, http://eotvos.dm.unipi.it/nobili/ggweb/ crandall

${ }^{22}$ A. M. Nobili et al., Class. Quantum Grav. 16, 1463 (1999).

${ }^{23}$ A. M. Nobili, G. L. Comandi, S. Doravari, D. Bramanti, E. Polacco, and F. Maccarrone (unpublished).

${ }^{24}$ This concept was originally introduced by G. C. Wigner and V. F. Weisskopf.

${ }^{25}$ G. L. Comandi, Ph.D. thesis, University of Pisa, 2004; http:// eotvos.dm.unipi.it/nobili/comandi_thesis 